\title{
Spectral Representations of Lorentz Invariant Distributions and Scale Transformation*
}

\author{
A. Rieckers and W. Güttinger \\ Department of Physics, University of Munich
}

Received June 15, 1967

\begin{abstract}
An approach to the theory of Lorentz invariant distributions is developed in terms of covariant spectral representations. The behaviour of singular invariant distributions under a change of scale is analyzed. It is shown that the conventional extension of homogeneous singular functions into distributions in $R^{4}$, followed by a breakdown of homogeneity, is incomplete. Homogeneous extensions depending on an arbitrary scaling parameter are introduced, calculation techniques are developed and various formulae having applications in quantum field theory are derived.
\end{abstract}

\section{Introduction}

The aim of this paper is to present a new approach to Lorentz invariant distributions in terms of spectral representations which exhibit the covariant form of the functionals, permit to overcome the "origin of the light cone" difficulties and lead to a considerable simplification of calculation techniques. Thus, the approach is possibly simpler than that of Refs. [1]-[5]. On the basis of this formalism we investigate the behaviour of certain singular invariant distributions under a change of scale: The conventional way of associating distributions in $R^{4}$ with homogeneous singular functions by regularization gives rise to functionals which are no longer homogeneous. Such inhomogeneous distributions (e.g. the propagators $\left(x^{2}-i 0\right)^{-n}, n \geqq 2$ ) are physically unacceptable because the space-time or momentum variables on which they depend carry dimension. The extension of singular homogeneous functions into homogeneous distributions in $R^{4}$ requires the introduction of an arbitrary scaling parameter bearing dimension. Then, a breakdown of dilatation symmetry by regularization can be avoided if a similarity transformation in space-time is accompanied by a corresponding change of this scaling parameter.

In Sec. 2 we develop the theory of Lorentz invariant distributions in terms of spectral representations. In Sec. 3 we analyze the problem

* Supported by the Deutsche Forschungsgemeinschaft. 
of extending distributions from a subspace to the entire $R^{4}$ in connection with scale transformations. Sec. 4 is devoted to a discussion of deltafunctions $\delta^{(n)}\left(x^{2}\right)$ etc. localized on the light cone and to a comparison of the various definitions appearing in the literature, all of them being summed up into an expression depending on the arbitrary scaling parameter. In Sec. 5 asymptotic and Laurent expansions are derived and the equivalence of certain extensions is proved. After a brief discussion of some special algebraically singular distributions in Sec. 6 we study in Sec. 7 Fourier transforms and analytic functionals in terms of spectral representations.

\section{Lorentz Invariant Distributions in Terms of Spectral Representations}

We denote by $R^{4}$ the four-dimensional Minkowski space of real points $x=\left(x_{v}\right)=\left(x_{0}, \mathbf{x}\right), \quad \mathbf{x}=\left(x_{1}, x_{2}, x_{3}\right)$, with the metric $x^{2}=x_{v}^{2}=x_{0}^{2}-\mathbf{x}^{2}$, $\mathbf{x}^{2}=\sum_{1}^{3} x_{i}^{2}$. When we write $f(x)$ for a distribution $f \in D^{\prime}\left(R^{4}\right)$ we merely wish to indicate that $f$ operates on test functions $\varphi(x)$ depending on $x \in R^{4} . f\langle\varphi\rangle$ denotes the value of the distribution at the element $\varphi(x)$ $=\varphi\left(x_{0}, \mathbf{x}\right)$.

Definition. A distribution $f \in \mathscr{D}^{\prime}\left(R^{4}\right)$ is said to be invariant under the restricted Lorentz group $L_{+}$if for any $\Lambda \in L_{+}^{\uparrow}$ the relation

$$
\Lambda f(x)\langle\varphi(x)\rangle=f(x)\langle\varphi(\Lambda x)\rangle=f(x)\langle\varphi(x)\rangle
$$

holds for all $\varphi \in \mathscr{D}\left(R^{4}\right)$.

Such $f$ will be called "invariant". In terms of the infinitesimal generators of $L_{+}^{\uparrow}, M_{0 k}=x_{0} \partial / \partial x_{k}+x_{k} \partial / \partial x_{0}, M_{i k}=x_{i} \partial / \partial x_{k}-x_{k} \partial / \partial x_{i}$, $(i, k=1,2,3) f$ is invariant only if $M_{0, k} f=M_{i, k} f=0$.

Definition. Let $\Lambda^{*}$ be an element of the antichronous component $L_{+}^{\downarrow}$. Then we define the reflected distribution $\bar{f}$ by

$$
\bar{f}=\Lambda^{*} f \text {. }
$$

For every invariant $f$, e.g. $\bar{f}(x)=f(-x)$, the definition (2.2) is independent of the particular choice of $\Lambda^{*}$. A distribution $f$ is called even if $\bar{f}=f$ and odd if $\bar{f}=-f$.

Every invariant distribution $f$ can be decomposed into an even part $f_{e}$ and an odd part $f_{0}$ according to

where

$$
f=f_{e}+f_{0}
$$

$$
f_{e}=\frac{1}{2}(f+\bar{f}), \quad f_{0}=\frac{1}{2}(f-\bar{f}) .
$$

From Ref. [6] we recall the following theorem. 
Theorem 1. Every invariant $f \in \mathscr{D}^{\prime}\left(R^{4}\right)$ whose support is the origin $x_{0}=\mathbf{x}=0$ has the form

$$
f=\sum_{0}^{N} c_{n} \square^{n} \delta(x)
$$

where $\delta(x)=\delta\left(x_{0}\right) \delta(\mathbf{x})$ and $\square=\partial^{2} / \partial x_{0}^{2}-\Delta$, with constant coefficients $c_{n}$ and a finite $N$. If $f \in \mathscr{Z}^{\prime}\left(C^{4}\right)$, then $N=\infty$ and $\varlimsup_{n \rightarrow \infty} \sqrt[n]{\left|c_{n}\right|(2 n) !}=0$.

Let $s \neq 0$ be a real number. Then the invariant hyperboloid $x^{2}=s$ is a regular surface in $R^{4}$. The light cone $x^{2}=0$ has the origin as a singular point. However, in the space $R^{4}-\mathbf{0}\left(R^{4}\right.$ minus the origin $\left.\mathbf{0}\right)$ the hyperboloids $x^{2}-s=0$ are regular surfaces for all $s \in R^{1}$. Hence, in $R^{4}-\mathbf{0}$ we can introduce a new local coordinate system $u=\left(u_{0}, u_{1}, u_{2}, u_{3}\right)$ with $u_{0}=x^{2}-s$ such that the Jacobian $D(x, u)$ remains different from zero. By starting from the formal integral $\int \delta^{(n)}\left(x^{2}-s\right) \varphi(x) d x$ and passing to the $u$ variables we obtain the following definition for $\delta^{(n)}\left(x^{2}-s\right)$ in $R^{4}-\mathbf{0}$ :

$$
\begin{gathered}
\delta\left(x^{2}-s\right)\langle\varphi(x)\rangle=\int \eta\left(0, u_{1}, u_{2}, u_{3}\right) d u_{1} d u_{2} d u_{3} \\
\delta^{(n)}\left(x^{2}-s\right)\langle\varphi(x)\rangle=\left.(-1)^{n} \int \frac{\partial^{n} \eta\left(u_{0}, u_{1}, u_{2}, u_{3}\right)}{\partial u_{0}^{n}}\right|_{u_{0}=0} d u_{1} d u_{2} d u_{3}
\end{gathered}
$$

where $\eta\left(u_{0}, \ldots, u_{3}\right)=\varphi(x(u)) D(x, u)$. We shall extend these definitions to the whole $R^{4}$ in Sec. 4 . (We call a distribution "defined in $R^{4}-\mathbf{0}$ " if it is defined on all testfunctions $\varphi(x)$ whose support does not contain the origin 0.)

For $s \geqq 0, \delta\left(x^{2}-s\right)$ can be decomposed in $R^{4}-\mathbf{0}$ according to

$$
\begin{aligned}
\delta\left(x^{2}-s\right) & =\left[\delta\left(x_{0}-\sqrt{\mathbf{x}^{2}+s}\right)+\delta\left(x_{0}+\sqrt{\mathbf{x}^{2}+s}\right)\right] / 2 \sqrt{\mathbf{x}^{2}+s} \\
& =: \theta\left(x_{0}\right) \delta\left(x^{2}-s\right)+\theta\left(-x_{0}\right) \delta\left(x^{2}-s\right)
\end{aligned}
$$

and this decomposition extends trivially to $R^{4}$. In $R^{4}$ we define

$$
\varepsilon\left(x_{0}\right) \delta\left(x^{2}-s\right)=\left\{\begin{array}{cl}
\theta\left(x_{0}\right) \delta\left(x^{2}-s\right)-\theta\left(-x_{0}\right) \delta\left(x^{2}-s\right), & s \geqq 0 \\
0 \quad, & s<0 .
\end{array}\right.
$$

With these definitions we can prove the following lemma.

Lomma. a) If $\phi \in \mathscr{D}\left(R^{4}-\mathbf{0}\right)$, then the meanvalue of $\phi$ over the hyperboloid $x^{2}-s=0$,

$$
\chi(s)=\delta\left(x^{2}-s\right)\langle\varphi(x)\rangle,
$$

is a test function of $\mathscr{D}\left(R^{1}\right)$.

b) If $\varphi \in D\left(R^{4}\right)$, then the function

$$
\chi_{+}(s)=\varepsilon\left(x_{0}\right) \delta\left(x^{2}-s\right)\langle\varphi(x)\rangle
$$

is a test function of $\mathscr{D}\left(R_{+}^{1}\right)=\mathscr{D}(s \geqq 0)$. 
Here $\mathscr{D}(s \geqq 0)$ is the space of all test functions $\chi_{+}(s)$ vanishing for $s<0$, having their supports bounded to the right and being infinitely differentiable for $s>0$, with right-sided derivatives at $s=0$.

Proof. a) Since $x^{2}-s=0$ is a regular surface in $R^{4}-0$ for all $s \in R^{1}$ at least one of the $\left|x_{\nu}\right|$ tends to infinity if $\left|x^{2}\right| \rightarrow \infty$. Since $\varphi$ has compact support, $\varphi(x) \equiv 0$ for $\left|x_{v}\right|>K^{\prime}>0$, and there exists a $K>0$ such that $\eta(u)=0$ for $s>K$, i.e., $\chi(s)=0$ for $|s|>K$. Furthermore, since $d \delta^{(n)}\left(x^{2}-s\right) / d s=-\delta^{(n+1)}\left(x^{2}-s\right)$ exists for all $n, \chi(s)$ is infinitely differentiable. Then $\chi \in D\left(R^{1}\right)$.

b) For $\varphi \in \mathscr{D}\left(R^{4}-\mathbf{0}\right)$ the same argument as in a) shows that $\chi_{+} \in \mathscr{D}\left(R_{+}^{1}\right)$. To demonstrate that $\chi_{+} \in \mathscr{D}\left(R_{+}^{1}\right)$ also for $\varphi \in \mathscr{D}\left(R^{4}\right)$ we note that the integrand in the following formula (obtained from (2.7) and (2.9))

$$
\begin{aligned}
& \varepsilon\left(x_{0}\right) \delta^{(n)}\left(x^{2}-s\right)\langle\varphi(x)\rangle=(-1)^{n} \int \frac{d^{n}}{d s^{n}}\left[\frac{\varphi\left(\sqrt{\mathbf{x}^{2}+s}, \mathbf{x}\right)-\varphi\left(-\sqrt{\mathbf{x}^{2}+s}, \mathbf{x}\right)}{2 \sqrt{\mathbf{x}^{2}+s}}\right] d \mathbf{x} \\
& \quad=(-1)^{n} \int \frac{d^{n}}{\left(2 x_{0} d x_{0}\right)^{n}}\left[\frac{\varphi\left(x_{0}, \mathbf{x}\right)-\varphi\left(-x_{0}, \mathbf{x}\right)}{2\left|x_{0}\right|}\right]_{x_{0}=\sqrt{\mathbf{x}^{2}+s}} d \mathbf{x}=(-1)^{n} \chi_{+}^{(n)}(s)
\end{aligned}
$$

is a regular and odd function of $x_{0}$ vanishing at $x_{0}=0$, i.e. at $|\mathbf{x}|=s=0$ the integral being convergent and the support of the resulting function is the one given in the Lemma.

The mapping of four dimensional testfunctions onto one dimensional test functions furnished by the Lemma enables us to set up spectral representations for invariant distributions which explicitly exhibit their invariance properties. To this end first let $f(s)$ be a locally summable function identifyable with a regular distribution. Then we can define the parametric integral

$$
\begin{aligned}
\left(\int_{-\infty}^{\infty} f(s) \delta\left(x^{2}-s\right) d s\right) & \langle\varphi(x)\rangle: \\
& =\int_{-\infty}^{\infty}\left(f(s) \delta\left(x^{2}-s\right)\right)\langle\varphi(x)\rangle d s=f(s)\langle\chi(s)\rangle
\end{aligned}
$$

since the $r h s$ exists for all $\varphi \in \mathscr{D}\left(R^{4}\right)$, taking into account that $f(s)$ is summable over all finite intervals. This leads to the following definition.

Definition. If $\delta\left(x^{2}-s\right)\langle\varphi(x)\rangle=\chi(s)$ is element of $\mathscr{D}\left(R^{1}\right)$ and $f(s) \epsilon$ $\in \mathscr{D}^{\prime}\left(R^{1}\right)$ is an arbitrary distribution, then the parametric "integral" is defined by

$$
\left(\int_{-\infty}^{\infty} f(s) \delta\left(x^{2}-s\right) d s\right)\langle\varphi(x)\rangle:=f(s)\langle\chi(s)\rangle .
$$

In case of an integrable $f(s)$ the $r h s$ of (2.13) reduces to that of (2.12).

It is appropriate to consider spectral representations for even and odd invariant distributions separately. 
Theorem 2. Every even invariant distribution $f_{e} \in \mathscr{D}^{\prime}\left(R^{4}-\mathbf{0}\right)$ has the spectrul representation

$$
f_{e}(x)\langle\varphi(x)\rangle=\int_{-\infty}^{\infty} f_{e}(s) \delta\left(x^{2}-s\right) d s\langle\varphi(x)\rangle
$$

where the spectral function (a distribution) $f_{e}(s) \in \mathscr{D}^{\prime}\left(R^{1}\right)$ is uniquely determined by $f_{e}(x)$.

In what follows we shall often omit the test functions $\varphi(x)$ in relations such as (2.14). The symbol $f_{e}(\cdots)$ in (2.14) obviously has two different meanings according to the argument.

Proof. Every $f_{e}(x) \in \mathscr{D}^{\prime}\left(R^{4}-\mathbf{0}\right)$ of the form (2.14) is even invariant. On the other hand, for every invariant $f_{e} \in \mathscr{D}^{\prime}\left(R^{4}-\mathbf{0}\right)$ we define the one dimensional distribution

$$
f_{e}(s)\langle\chi(s)\rangle:=f_{e}(x)\left\langle h(x) \chi\left(x^{2}\right)\right\rangle
$$

for every $\chi(s) \in \mathscr{D}\left(R^{1}\right) . h(x)$ is an infinitely differentiable auxiliary function whose support does not contain the origin and such that $\delta\left(x^{2}-s\right)\langle h(x)\rangle=1$ for all $s \in R^{1}$. One could choose the support of $h(x)$ in the way, that the support of $h(x) \chi\left(x^{2}\right)$ is bounded. The following arguments show that this is not necessary in virtue of $f_{e}(x)$ being Lorentz invariant. For the distribution $\hat{f}_{e}(x)$ defined by

we have

$$
\hat{f}_{e}(x):=\int_{-\infty}^{\infty} f_{e}(s) \delta\left(x^{2}-s\right) d s
$$

Hence,

$$
\begin{aligned}
\hat{f}_{e}(x)\langle\varphi(x)\rangle & =\int_{-\infty}^{\infty} f_{e}(s) \delta\left(x^{2}-s\right)\langle\varphi(x)\rangle d s \\
& =f_{e}(s)\langle\chi(s)\rangle=f_{e}(x)\left\langle h(x) \chi\left(x^{2}\right)\right\rangle .
\end{aligned}
$$

$$
\left(\hat{f}_{e}(x)-f_{e}(x)\right)\langle\varphi(x)\rangle=f_{e}(x)\langle\omega(x)\rangle
$$

where $\omega(x)=h(x) \chi\left(x^{2}\right)-\varphi(x)$ and $\delta\left(x^{2}-s\right)\langle\omega(x)\rangle=0$ for all $s$.

Let us decompose the space $R^{4}$ into overlapping bounded domains $G_{k}$ with the following properties: $G_{0}$ contains the origin but does not intersect with the support of $\omega(x)$. (This is possible since the support of $\omega(x)$ does not contain the origin and is closed by definition). The domains $G_{k}, k=1,2, \ldots$, are supposed not to contain the origin. Thus we can choose an $l=l(k)$ with $x_{l} \neq 0$ in $G_{k}$. Let $\left\{e_{k}\right\}$ be the partition of the unity belonging to $\left\{G_{k}\right\}$ and $\omega_{k}(x):=\omega(x) e_{k}(x)$. Only a finite number of the $\omega_{k}(x)$ are not identically zero. Then we have

$$
\delta\left(x^{2}-s\right)\langle\omega(x)\rangle=\sum_{k} \int_{G_{k} \cap\left(x^{2}-s\right)=0} \frac{\omega_{k}(x)}{2\left|x_{i}\right|} d \mathbf{x}_{i}
$$

where $i(k) \neq l(k)$. The $x_{l}$ have to be expressed in terms of the new 
variables $u_{l}=x^{2}-s$ and $u_{i}=x_{i}$. In $G_{k}$ we write

$$
\omega_{k}(x) / 2\left|x_{l}\right|=\sum_{i} \partial \sigma_{k i}(x) / \partial x_{i}
$$

with infinitely differentiable $\sigma_{k}$. Transforming the 3 -dimensional volume integrals of (2.15) into 2-dimensional surface integrals one deduces from $\delta\left(x^{2}-s\right)\langle\omega(x)\rangle=0$ for all $s$ that the $\sigma_{k l}$ have bounded supports. On the other hand, in the old variables we have

$$
\omega_{k}=\sum_{i} 2 M_{l i} \sigma_{k i}
$$

where the $M_{l i}$ are the generators of $L_{+}^{\uparrow}$. Thus

$$
f_{e}(x)\langle\omega(x)\rangle=2 \sum_{k, i(k)} M_{l(k) i(k)} f_{e}(x)\left\langle\sigma_{k i}(x)\right\rangle=0
$$

in virtue of the invariance of $f_{e}(x)$.

Thus $f_{e}(s)$ is uniquely determined by $f_{e}(x)$ and independent of the auxiliary function $h(x)$. This completes the proof.

If $\varphi(x) \in \mathscr{D}\left(R^{4}\right)$ then $\chi(s)=\delta\left(x^{2}-s\right)\langle\varphi(x)\rangle$ has the form

$$
\chi(s)=h_{1}(s)+h_{2}(s) \log s
$$

where $h_{1}, h_{2} \in \mathscr{D}\left(R^{1}\right)$ and $h_{2}(0)=0$ (cf. also Sec. 5). As shown in Ref. [4] the space $\mathscr{H}\left(R^{1}\right)$ consisting of all functions of type (2.17) can be equipped with a topology. Denoting its dual by $\mathscr{H}^{\prime}\left(R^{1}\right)$ one has the following theorem.

Theorem 3. Every even invariant distribution $f_{e}(x) \in \mathscr{D}^{\prime}\left(R^{4}\right)$ admits the spectral representation

$$
f_{e}(x)=\int_{-\infty}^{\infty} f_{e h}(s) \delta\left(x^{2}-s\right) d s+\sum_{0}^{N} c_{n} \square^{n} \delta(x)
$$

where $f_{e h}(s)$ is element of $\mathscr{H}^{\prime}\left(R^{1}\right) . f_{e h}$ is not uniquely determined by $f_{e}(x)$ : The "spectral function" $f_{e h}(s)$ can be altered if simultaneously the coefficients $c_{n}$ are changed. Only the restriction $f_{e}(s)$ of $f_{e h}(s)$ to $\mathscr{D}^{\prime}\left(R^{1}\right)$ is uniquely determined according to Theorem 2.

By means of the second part of the previous Lemma one proves in analogy with Theorem 2 the following theorem.

Theorem 4. Every odd invariant distribution $f_{0}(x) \in \mathscr{D}^{\prime}\left(R^{4}\right)$ has the spectral representation

$$
f_{0}(x)=\int_{0}^{\infty} f_{0}(s) \varepsilon\left(x_{0}\right) \delta\left(x^{2}-s\right) d s
$$

where the spectral function $f_{0}(s) \in \mathscr{D}^{\prime}(s \geqq 0)$ is uniquely determined by $f_{0}(x) \cdot \mathscr{D}^{\prime}(s \geqq 0)$ is the dual to $\mathscr{D}(s \geqq 0)$ and is isomorphic to the subspace $\mathscr{D}^{\prime}+$ of all those distributions of $\mathscr{D}^{\prime}\left(R^{1}\right)$ whose support is contained in $s \geqq 0$. 
Let us summarize the preceding results into

Theorem 5. Every invariant distribution $f(x) \in \mathscr{D}^{\prime}\left(R^{4}\right)$ has the spectral representation

$f(x)=\int_{-\infty}^{\infty} f_{e h}(s) \delta\left(x^{2}-s\right) d s+\int_{0}^{\infty} f_{0}(s) \varepsilon\left(x_{0}\right) \delta\left(x^{2}-s\right) d s+\sum_{0}^{N} c_{n} \square^{n} \delta(x)$

where $f_{e h} \in \mathscr{H}^{\prime}\left(R^{1}\right)$. $f_{e h}$ has a uniquely determined restriction to $\mathscr{D}^{\prime}\left(R^{1}\right)$ and $f_{0}$ is an uniquely determined distribution of $\mathscr{D}^{\prime}(s \geqq 0) . N$ depends on the order of $f(x)$.

In virtue of the above spectral representations the following symbolic notation is justified. For every even invariant distribution $f_{e}(x)$ we shall write

$$
f_{e}(x):=f\left(x^{2}\right)
$$

and for every odd invariant distribution we set ${ }^{1}$

$$
f_{0}(x):=\varepsilon\left(x_{0}\right) f\left(x^{2}\right) .
$$

Let now $f(s)$ be a given distribution in $\mathscr{H}^{\prime}(s \geqq 0)$. Considering $f(s)$ as a spectral function we can construct the following distributions:

$$
\begin{gathered}
\theta\left(x^{2}\right) f\left(x^{2}\right):=\int_{-\infty}^{\infty} f(s) \delta\left(x^{2}-s\right) d s:=\int_{0}^{\infty} f(s) \delta\left(x^{2}-s\right) d s \\
\varepsilon\left(x_{0}\right) f\left(x^{2}\right):=\int_{0}^{\infty} f(s) \varepsilon\left(x_{0}\right) \delta\left(x^{2}-s\right) d s \\
\theta\left( \pm x_{0}\right) f\left(x^{2}\right):=\frac{1}{2}\left[\theta\left(x^{2}\right) f\left(x^{2}\right) \pm \varepsilon\left(x_{0}\right) f\left(x^{2}\right)\right] \\
=\int_{0}^{\infty} f(s) \theta\left( \pm x_{0}\right) \delta\left(x^{2}-s\right) d s \\
\theta\left(-x^{2}\right) f\left(x^{2}\right):=\int_{0}^{\infty} f(-s) \delta\left(x^{2}+s\right) d s .
\end{gathered}
$$

In (2.22) to (2.26) the products of the distributions with step functions are merely symbolic notations for the well defined quantities on the right hand side. If $f(s)$ is a given singular function and one wants to construct an invariant distribution with prescribed support properties having $f(s)$ as spectral function the above formulas suggest the following procedure: Extend first $f(s)$ to $\mathscr{D}^{\prime}\left(R^{1}\right)$, perform a second extension to $\mathscr{H}^{\prime}\left(R^{1}\right)$ and apply then the spectral representation. In the following section more direct extension procedures are discussed.

1 In (2.22), (2.25) the l.h.s. should be "multiplied" by $\theta\left(\mathrm{x}^{2}\right)$. Since, however, these distributions are Lorentz invariant such factor can be omitted. We shall adhere to this convenience in all what follows. 
Remark. We have introduced a mapping of invariant four dimensional distributions onto one dimensional distributions by means of spectral representations. The same mapping can be generated through a transformation of variables. Let the support of $\varphi(x)$ be contained in the region $x_{0}>0$ of $R^{4}$. Introducing the transformation $T x=u: u_{0}=x^{2}, u_{i}=x_{i}$, we define

$$
\begin{aligned}
(T f)(u)\langle\varphi(u)\rangle & =f(x)\langle\varphi(T x)|\partial T x| \partial x \mid\rangle \\
& =f(x)\left\langle\varphi\left(x^{2}, x_{1}, x_{2}, x_{3}\right) 2 x_{0}\right\rangle .
\end{aligned}
$$

As in the proof of Theorem 2 we find that

$$
\left(\partial(T f) / \partial u_{i}\right)\langle\varphi(u)\rangle=2 M_{o i} f(x)\left\langle\varphi\left(x^{2}, \mathrm{x}\right)\right\rangle=0
$$

for $i=1,2,3$. Thus, $T f$ is constant in the variables $u_{1}, u_{2}, u_{3}$ and we can write it as a direct product:

$$
(T f)(u)=f\left(u_{0}\right) \times 1_{\mathbf{u}} .
$$

Hence in the domain where $T$ is a regular transformation (cf. [6]) it provides a mapping of $f(x)$ onto a one dimensional distribution $f\left(u_{0}\right)$. If $T$ is singular (what happens if the origin is included) an extension procedure of the type discussed in the next section is to be superimposed.

\section{Extension of Invariant Distributions to $\boldsymbol{R}^{4}$ and Scale Transformation}

Two attitudes may be taken towards the construction of singular invariant distributions in $R^{4}$. On the one hand, a classical function singular on the cone may be associated with a distribution defined on a certain subspace of $\mathscr{D}\left(R^{4}\right)$ and then be extended to the whole $\mathscr{D}\left(R^{4}\right)$. Or else, singular distributions may be generated entirely within the distribution frame by differentiating regular distributions or by analytic continuation in a parameter.

\subsection{Extensions Depending on a Scaling Parameter}

Let $f(x)$ be an invariant distribution defined on a subspace $\mathscr{B} \subset \mathscr{D}\left(R^{4}\right)$. For example $\mathscr{B}$ may be identical with the set of test functions having the property $\varphi^{(v)}(0)=0$ for $v \leqq N$, or may consist of all those test functions such that $\chi(s)=\delta\left(x^{2}-s\right)\langle\varphi(x)\rangle$ vanishes sufficiently strongly at $s=0$. Let $\mathbf{E} f$ be a given extension of $f$ to $\mathscr{D}\left(R^{4}\right)$. We consider the dilatation operator $\mathbf{U}_{a}$, with $a=\left(a_{1}, a_{2}, a_{3}, a_{4}\right)$, acting on a distribution $g(x)$ according to

$$
\left(\mathbf{U}_{a} g\right)(x)\langle\varphi(x)\rangle:=g(x)\left\langle\varphi(x / a)\left|\underset{0}{1 / I I a_{i}}\right|\right\rangle
$$

where $x / a=\left(x_{0} / a_{0}, \ldots, x_{3} / a_{3}\right)$. We confine ourselves to dilatations which affect all coordinates $x$ in the same way, i.e. we put $a=(a, a, a, a)$, 14 Commun. math. Phys., Vol. 7 
where $a>0$ is a constant. (There would be no problem in admitting scaling transformations depending on $x$. In that case one had to require that $a(x)>0$ is infinitely differentiable such that $a(x)$ and $\log a(x)$ are multipliers.) $\mathbf{U}_{a}$ is well defined in $\mathscr{B}^{\prime}$ and $\mathscr{D}^{\prime}$. In general, however, the extension procedure does not commute with the dilatation, i.e.

$$
\mathbf{U}_{a} \mathbf{E} f \neq \mathbf{E} \mathbf{U}_{a} f
$$

In particular, if $f(x)$ is homogeneous in the subspace $\mathscr{B}$ the extension $\mathbf{E} f$ in general is no longer homogeneous. Let us then introduce a class of extensions $\mathbf{E}_{a} f$ depending on a parameter $a$ defined by

$$
\mathbf{E}_{a} f:=\mathbf{U}_{a}^{-1} \mathbf{E} \mathbf{U}_{a} f \text {. }
$$

By definition $\mathbf{E}_{1} f=\mathbf{E} f$ and $\mathbf{E}_{a} f$ is independent of $a$ if and only if the extension commutes with the dilatation. The group property of $\mathbf{U}_{a}$ implies that

or

$$
\mathbf{U}_{a^{\prime}}^{-1} \mathbf{E}_{a} \mathbf{U}_{a^{\prime}} f=\mathbf{E}_{a a^{\prime}} f
$$

$$
\mathbf{U}_{a^{\prime}} \mathbf{E}_{a a^{\prime}} f=\mathbf{E}_{a} \mathbf{U}_{a^{\prime}} f
$$

Thus by introducing an $a$-dependent extension we have reestablished "commutativity" of extension and dilatation if a dilatation of $x$ is accompanied by a corresponding dilatation of $a$. In particular if $f(x)$ is homogeneous of degree $\lambda$ in $\mathscr{B}$ we have (cf., e.g. (4.13), (6.6))

$$
\mathbf{U}_{a^{\prime}} \mathbf{E}_{a a^{\prime}} f=a^{\prime \lambda} \mathbf{E}_{a} f,
$$

i.e. homogeneity is preserved also after the extension if $a$ is subject to the same dilatation as $x$. We call distributions which are homogeneous on a subspace $\mathscr{B}$ and whose extension is $a$-dependent "pseudo-homogeneous". Pseudo-homogeneous distributions are in fact associated generalized functions (associated to the operator $\mathbf{U}_{a}$, cf. [5]). Now, if both $a$ and $x$ are numerical ratios to the same physical unit, a change in scale of $x$ is accompanied by the corresponding change in scale of $a$. Furthermore we shall see later that pseudo-homogeneous distributions of rational degree of homogeneity (whose argument may bear a dimension on the subspace $\mathscr{B}$ ) are dimensionally correct on $\mathscr{D}\left(R^{4}\right)$ only if we ascribe $a$ the same dimension as $x$.

If $\hat{\mathbf{E}} f$ is another extension of $f$ and if $\hat{\mathbf{E}} f=\mathbf{E}_{a_{0}} f$ for a special $a_{0}$ we have in virtue of (3.2)

$$
\widehat{\mathbf{E}}_{a} f=\mathbf{E}_{a a_{0}} f .
$$

The meaning of $\mathbf{E} \mathbf{U}_{a} f$ in the general definition of $\mathbf{E}_{a} f$ in (3.1) can most easily be understood in the one dimensional case. Let $f(s)$ be defined on the subspace $\mathscr{B}$ of those test functions $\varphi(s)$ which vanish at the origin $s=0$ with its first $N$ derivatives. Then the most general extension 
E $f$ of $f$ to $\mathscr{D}^{\prime}\left(R^{1}\right)$ is given by

with

$$
(E f)(s)\langle\psi(s)\rangle=f(s)\langle\varphi(s)\rangle+\sum_{0}^{N} \psi^{(n)}(0) c_{n}
$$

$$
\varphi(s)=\psi(s)-\frac{\sum_{0}^{N}}{0} \psi^{(n)}(0) \varphi_{n}(s) ; \quad \varphi_{n}^{(\nu)}(0)=\delta_{n v} ; \quad n, v=0,1, \ldots, N
$$

and $c_{n}=(\mathbf{E} f)\left\langle\varphi_{n}\right\rangle$.

Conversely, with an arbitrarily given set of $c_{n}$ and $\varphi_{n}(s)$ satisfying (3.5) one can construct an extension of $f$ from $\mathscr{B}^{\prime}$ to $\mathscr{D}^{\prime}$ according to (3.5). By definition of $\mathbf{U}_{a}$,

$$
\begin{aligned}
& \left(\mathbf{U}_{a} \mathbf{E} f\right)\langle\psi(s)\rangle=(\mathbf{E} f)(s)\left\langle\psi(s / a)|a|^{-1}\right\rangle \\
& =f(s)\left\langle\psi(s / a) a^{-1}-\sum_{0}^{N} a^{-n-1} \psi^{(n)}(0) \varphi_{n}(s)\right\rangle+\sum_{0}^{N} a^{-n-1} \psi^{(n)}(0) c_{n} .
\end{aligned}
$$

It seems natural to define $\mathbf{E} \mathbf{U}_{a} f$ such that the dilatation operates only in the subspace $\mathscr{B}$, i.e.

$$
\begin{aligned}
\left(\mathbf{E} \mathbf{U}_{a} f\right) & (s)\langle\psi(s)\rangle:=f(s)\left\langle\varphi(s / a) a^{-1}\right\rangle+\sum_{0}^{N} \psi^{(n)}(0) c_{n} \\
& =f(s)\left\langle\psi(s / a) a^{-1}-a^{-1} \sum_{0}^{N} \psi^{(n)}(0) \varphi_{n}(s / a)\right\rangle+\sum_{0}^{N} \psi^{(n)}(0) c_{n} .
\end{aligned}
$$

Eqs. (3.6) and (3.7) coincide if and only if we can choose $\varphi_{n}(s)=s^{n} / n$ ! and set $(E f)(s)\left\langle s^{n} \mid n !\right\rangle=c_{n}=0$. These special $\varphi_{n}(s)$ have no longer compact support. In case that $f(s)\langle\psi(s)\rangle$ exhibits logarithmic divergencies, and only then, we cannot choose $\varphi_{N}(s)$ equal to $s^{N} / N$ !. Thus no exteusion Ef exists which is independent of the scaling parameter $a$. The requirement that an extension $\mathbf{E} f$ of $f$ commutes with dilatation, determines uniquely this extension and is apparently equivalent with Gelfand's canonical regularization [5].

The same considerations are valid in $\mathscr{H}^{\prime}\left(R^{1}\right)$ and, therefore, hold for invariant distributions too. We shall see that $a$-dependent invariant extensions depend on $a^{2}$ only. If $x$ is the momentum space variable $a^{2}$ has dimension of mass squared. Examples demonstrate that $a$ stands exactly at the place a cut-off would stand in a singular quantum field theory. However, $a$ actually is not a cutoff, the theory remaining strictly local. $a$ might be viewed as an arbitrary finite factor which can be split off from any cut-off $K: K=a K^{\prime} ; K, K^{\prime} \rightarrow \infty$ in the local limit.

\subsection{Constructive Forms of Invariant Extensions}

Let $f(s)$ be a function with an algebraic singularity at $s=0$ and locally summable for $s>0$. The invariant distribution

$$
\theta\left(x_{0}\right) f\left(x^{2}\right):=\int_{0}^{\infty} f(s) \theta\left(x_{0}\right) \delta\left(x^{2}-s\right) d s
$$


is defined only on those test functions $\varphi(x)$ whose meanvalue $\chi(s)$ $=\delta\left(x^{2}-s\right)\langle\varphi(x)\rangle$ vanishes sufficiently strongly at $s=0$. We consider three possibilities of extending this functional to the whole $\mathscr{D}^{\prime}\left(R^{4}\right)$.

The first of these is given by the construction (cf. [6])

$$
\begin{aligned}
\mathbf{E}_{a}\left\{\theta\left(x_{0}\right) f\left(x^{2}\right)\right\}: & =0\left(x_{0}\right) f_{a}\left(x^{2}\right) \\
& =\operatorname{Res}_{z=0}\left[\frac{1}{z} \int_{0}^{\infty} f(s)\left(\frac{a^{2}}{s}\right)^{z} \theta\left(x_{0}\right) \delta\left(x^{2}-s\right) d s\right] .
\end{aligned}
$$

Here one has first to perform the $s$-integration for $\operatorname{Re}(z)$ sufficiently large negative, then to continue the resulting function of $z$ analytically to $z=0$ and finally to apply the residue (which projects out the pole-free part).

Another extension of $\theta f$ consists in taking the finite part in the sense of Hadamard, viz.,

$$
\begin{aligned}
\hat{\mathbf{E}}_{a}\left\{\theta\left(x_{0}\right) f\left(x^{2}\right)\right\} & :=\operatorname{Pf}\left\{\theta\left(x_{0}\right) f_{a}\left(x^{2}\right)\right\}=\operatorname{Pf}_{\varepsilon \rightarrow 0} \int_{a^{2} \varepsilon}^{\infty} f(s) \theta\left(x_{0}\right) \delta\left(x^{2}-s\right) d s \\
& =\lim _{\varepsilon \rightarrow 0}\left[\int_{a^{2} \varepsilon}^{\infty} f(s) \theta\left(x_{0}\right) \delta\left(x^{2}-s\right) d s-I_{a, \varepsilon}(x)\right]
\end{aligned}
$$

where $I_{a, \varepsilon}(x)$ - the "infinite part" of the integral - is by definition of the form

$$
I_{a, \varepsilon}(x)=\sum_{\mu, \nu}^{M T, N} c_{\mu \nu}^{a}(x) \varepsilon^{\mu} \log ^{\nu} \varepsilon
$$

with $c_{\mu \nu}^{a}(x) \in \mathscr{D}^{\prime}\left(R^{4}\right), \operatorname{Re} \mu \leqq 0, v$ a nonnegativ integer, and $\mu, v$ not simultaneously equal zero. $I_{a, \varepsilon}(x)$ is - if it exists - uniquely determined by the requirement that the limit in (3.9) is finite. Therefore $\operatorname{Pf}\left\{\theta\left(x_{0}\right) f_{a}\left(x^{2}\right)\right\}$ is also uniquely determined. The integral in (3.9) is a distributionvalued function of $\varepsilon$. The analogous definition of Pf holds for a numbervalued function $f(\varepsilon)$.

We shall see in Sec. 5 that (3.8) and (3.9) yield the same distributions. Their $a$-dependence is an immediate consequence of Definition 3.1.

\subsection{Differentiation of Invariant Distributions}

Finally one can construct invariant extensions by operating with invariant differential operators on regular invariant distributions. For two times differentiable functions $g\left(x^{2}\right)$ we have (via the chain rule)

$$
\square g\left(x^{2}\right)=\left(D_{u} g(u)\right)_{u=x^{2}}
$$

where $D_{u}$ and its adjoint $D_{u}^{+}$are given by

$$
D_{u}=4 u \frac{d^{2}}{d u^{2}}+8 \frac{d}{d u}, \quad D_{u}^{+}=4 u \frac{d^{2}}{d u^{2}} .
$$


The chain rule holds also for $\delta^{(n)}\left(x^{2}-s\right)$ in $R^{4}-\mathbf{0}$ whre $x^{2}-s=0$ is a regular surface (cf. [5]). Therefore we have in $R^{4}-\mathbf{0}$

By means of

$$
\square \delta\left(x^{2}-s\right)=\left[D_{u} \delta(u-s)\right]_{u=x^{2}} .
$$

$$
x^{2} \delta^{(n)}\left(x^{2}-s\right)=s \delta^{(n)}\left(x^{2}-s\right)-n \delta^{(n-1)}\left(x^{2}-s\right)
$$

it follows that

$$
\begin{gathered}
\square \delta\left(x^{2}-s\right)=D_{s}^{+} \delta\left(x^{2}-s\right) \\
\square \delta^{(n)}\left(x^{2}-s\right)=4 s \delta^{(n+2)}\left(x^{2}-s\right)-4 n \delta^{(n+1)}\left(x^{2}-s\right) .
\end{gathered}
$$

Equs. (3.12)-(3.14) hold in $R^{4}$ for $s \neq 0$, in $R^{4}-0$ for all $s$.

Let $g(s)$ be locally summable and two times differentiable for $s>0$. Define

$$
\theta\left(x_{0}\right) g\left(x^{2}\right)=\int_{0}^{\infty} g(s) \theta\left(x_{0}\right) \delta\left(x^{2}-s\right) d s .
$$

By means of (3.12) we obtain

$$
\begin{array}{r}
\square\left\{\theta\left(x_{0}\right) g\left(x^{2}\right)\right\}=\lim _{\varepsilon \rightarrow 0} \int_{\varepsilon}^{\infty} g(s) D_{s}^{+} 0\left(x_{0}\right) \delta\left(x^{2}-s\right) d s \\
=4 \lim _{\varepsilon \rightarrow 0}\left[\varepsilon g(\varepsilon) \theta\left(x_{0}\right) \delta^{\prime}\left(x^{2}-\varepsilon\right)+\frac{d}{d \varepsilon}(\varepsilon g(\varepsilon)) \theta\left(x_{0}\right) \delta\left(x^{2}-\varepsilon\right)+\right. \\
\left.+\int_{\varepsilon}^{\infty} \frac{d^{2}}{d s^{2}}(s g(s)) \theta\left(x_{0}\right) \delta\left(x^{2}-s\right) d s\right] .
\end{array}
$$

Although $4 d^{2}(s g(s)) / d s^{2}=f(s)$ in general is not summable, the limit in (3.15) exists since every distribution is infinitely differentiable. Therefore (3.15) furnishes an extension of $\theta\left(x_{0}\right) f(s)$. By repeated differentiation one can construct more singular distributions.

All what had been said about distributions of type $\theta\left(x_{0}\right) f\left(x^{2}\right)$ is immediately extended to distributions $\theta\left(-x_{0}\right) f\left(x^{2}\right), \theta\left( \pm x^{2}\right) f\left(x^{2}\right)$ and to linear combinations of these.

Remark. Defining $\theta\left(x_{0}-\varepsilon\right) g\left(x^{2}\right):=\int_{\varepsilon}^{\infty} g(s) \theta\left(x_{0}\right) \delta\left(x^{2}-s\right) d s$, we can write

$$
\begin{aligned}
& \square\left\{\theta\left(x_{0}-\varepsilon\right) g\left(x^{2}\right)\right\}\langle\varphi(x)\rangle \\
& =4\left[\varepsilon g(\varepsilon) \theta\left(x_{0}\right) \delta^{\prime}\left(x^{2}-\varepsilon\right)+\frac{d}{d \varepsilon}(\varepsilon g(\varepsilon)) \theta\left(x_{0}\right) \delta\left(x^{2}-\varepsilon\right)+\right. \\
& \left.\quad+\int_{\varepsilon}^{\infty} \frac{d^{2}}{d s^{2}}(s g(s)) \theta\left(x_{0}\right) \delta\left(x^{2}-s\right) d s\right]\langle\varphi(x)\rangle
\end{aligned}
$$


in the form

$$
\begin{aligned}
\int_{V_{+}^{\varepsilon}} g\left(x^{2}\right) \square \varphi(x) d x= & \int_{V_{+}^{\varepsilon}} \varphi(x) \square g\left(x^{2}\right) d x+ \\
& \quad+\int_{x^{2}=\varepsilon}\left[\varphi(x)-\frac{\partial g\left(x^{2}\right)}{\partial n}-g\left(x^{2}\right) \frac{\partial \varphi(x)}{\partial n}\right] d \sigma_{\varepsilon},
\end{aligned}
$$

which is just Green's formula for the region $V_{+}^{\varepsilon}=\left\{x^{2} \geqq \varepsilon, x_{0}>0\right\}$ (cf. $[6])$.

In $R^{4}-\mathbf{0}$ we have a general formula for the invariant derivative. By means of $(3.12 \mathrm{a})$ and the spectral representation we have the relation

$\square f(x)=\int_{-\infty}^{\infty}\left(D_{s} f_{e}(s)\right) \delta\left(x^{2}-s\right) d s+\int_{0}^{\infty}\left(D_{s} f_{0}(s)\right) \varepsilon\left(x_{0}\right) \delta\left(x^{2}-s\right) d s$.

Here the derivatives are to be taken in the sense of distributions and therefore derivatives of $\delta(s)$ may appear in the integrand. (3.16) remains valid for the odd part of $f(x)$ in the whole $R^{4}$.

If $f(s)$ has an algebraic singularity at $s=0$ we determine $\square\left\{\theta\left(x_{0}\right) f\left(x^{2}\right)\right\}$ for the two extensions defined in Sec. 3.2:

With the residue procedure we obtain in $R^{4}$

$$
\begin{aligned}
\square\left\{\theta\left(x_{0}\right) f_{a}\left(x^{2}\right)\right\} & =\operatorname{Res}_{z=0}\left[\frac{1}{z} \int_{0}^{\infty} f(s)\left(\frac{a^{2}}{s}\right)^{z} D_{s}^{+} \theta\left(x_{0}\right) \delta\left(x^{2}-s\right) d s\right] \\
& =4 \operatorname{Res}_{z=0}\left[\frac{1}{z} \int_{0}^{\infty} \frac{d^{2}}{d s^{2}}\left[(s f(s))\left(\frac{a^{2}}{s}\right)^{z}\right] \theta\left(x_{0}\right) \delta\left(x^{2}-s\right) d s\right] .
\end{aligned}
$$

The last equation holds for sufficiently large negative values of $\operatorname{Re}(z)$ such that the boundary terms coming from the partial integration vanish and, therefore, holds also for $z=0$ by analytic continuation. Similarly,

$$
\square\left\{\theta\left(-x^{2}\right) f_{a}\left(x^{2}\right)\right\}=-4 \operatorname{Res}_{z=0}\left[\frac{1}{z} \int_{0}^{\infty} \frac{d^{2}}{d s^{2}}\left[s f(-s)\left(\frac{a^{2}}{s}\right)^{z}\right] \delta\left(x^{2}+s\right) d s\right] .
$$

By means of the Pf concept we derive

$$
\square\left\{\operatorname{Pf}\left[\theta\left(x_{0}\right) f_{a}\left(x^{2}\right)\right]\right\}=\lim _{\varepsilon \rightarrow 0}\left[\int_{a^{2} \varepsilon}^{\infty} f(s) D_{s}^{+} \theta\left(x_{0}\right) \delta\left(x^{2}-s\right) d s-\square I_{a, \varepsilon}(x)\right]
$$

whence, integrating by parts,

$$
\begin{aligned}
\square\left\{\operatorname{Pf}\left[\theta\left(x_{0}\right) f_{a}\left(x^{2}\right)\right]\right\}= & 4 \operatorname{Pf}_{\varepsilon \rightarrow 0}\left[a^{2} \varepsilon f\left(a^{2} \varepsilon\right) \theta\left(x_{0}\right) \delta^{\prime}\left(x^{2}-a^{2} \varepsilon\right)\right]+ \\
& +4 \operatorname{Pf}_{\varepsilon \rightarrow 0}\left[\frac{d}{d \varepsilon}\left(\varepsilon f\left(a^{2} \varepsilon\right)\right) \theta\left(x_{0}\right) \delta\left(x^{2}-a^{2} \varepsilon\right)\right]+ \\
& +4 \operatorname{Pf}_{\varepsilon \rightarrow 0}\left[\int_{a^{2} \varepsilon}^{\infty} \frac{d^{2}}{d s^{2}}(s f(s)) \theta\left(x_{0}\right) \delta\left(x^{2}-s\right) d s\right] .
\end{aligned}
$$

The results of Sec. 5 show that (3.17) and (3.19) are equivalent. 


\section{Theory of Delta-functions}

By means of the spectral representations of Sec. 2 all operations on Lorentz invariant distributions are shifted to the delta-functions. Thus, the theory of the latter, especially, the asymptotic expansion of $\delta\left(x^{2}-s\right)$ at $s=0$ and the extension of the $\delta^{(n)}\left(x^{2}\right)$-functions from $R^{4}-\mathbf{0}$ to the whole of $R^{4}$ deserves particular attention.

According to (2.8) we have for $s>0$

$$
\begin{aligned}
\theta\left(x_{0}\right) \delta^{(n)}\left(x^{2}-s\right)\langle\varphi(x)\rangle & =(-1)^{n} \int_{-\infty}^{\infty} \frac{d^{n}}{d s^{n}}\left[\frac{\varphi\left(\sqrt{\mathbf{x}^{2}+s, \mathbf{x}}\right)}{2 \sqrt{\mathbf{x}^{2}+s}}\right] d \mathbf{x} \\
& =2 \pi(-1)^{n} \int_{0}^{\infty} \frac{d^{n}}{d s^{n}}\left[\frac{\Phi\left(\sqrt{r^{2}+s}, r^{2}\right)}{\sqrt{r^{2}+s}}\right] r^{2} d r
\end{aligned}
$$

where $4 \pi \Phi\left(\sqrt{r^{2}+s, r^{2}}\right)=\int_{\mathbf{x}^{2}} \varphi\left(\sqrt{\mathbf{x}^{2}}+s, \mathbf{x}\right) d \Omega$ is the meanvalue of $\varphi(x)$ over the sphere of radius $r$. The integral (4.1) diverges as $s$ tends to zero. It has been shown by Mexнée [2] that

$$
\begin{aligned}
\operatorname{Pif}\left\{\theta\left(x_{0}\right) \delta^{\prime}\left(x^{2}-s\right)\right\} & =-\frac{\pi}{2} \delta(x) \log s \\
\operatorname{Pif}\left\{\theta\left(x_{0}\right) \delta^{(n)}\left(x^{2}-s\right)\right\} & =\sum_{\nu=0}^{n-2} c_{\nu}(x) s^{-n+\nu+1}+c_{n-1}(x) \log s,
\end{aligned}
$$

where Pif is that part of (4.1) which diverges as $s \rightarrow 0$ ("partie infinie"). Since $\theta\left(x_{0}\right) \delta^{(n)}\left(x^{2}\right)$ exhibits no divergencies in $R^{4}-\mathbf{0}$ the $c_{\nu}(x)$ are invariant distributions localized at the origin.

From (4.2) it follows that $\theta\left(x_{0}\right) \delta\left(x^{2}-s\right)$ posses an asymptotic expansion of arbitrary precision in the function system $s^{\mu} \log ^{v} s$ of the following general form

$$
\begin{aligned}
\theta\left(x_{0}\right) \delta\left(x^{2}-s\right) \sim \theta\left(x_{0}\right) a_{0}(x) & +\sum_{n=1}^{\infty}\left[\theta\left(x_{0}\right) a_{n}(x) s^{n}+\right. \\
& \left.+b_{n-1}(x) s^{n} \log s\right]
\end{aligned}
$$

Here $\theta\left(x_{0}\right) a_{n}(x)$ is a symbolic notation of a well determined distribution. By differentiating (4.3) $n$ times with respect to $s$ one obtains

$$
\begin{aligned}
& (-1)^{n} \theta\left(x_{0}\right) \delta^{n}\left(x^{2}-s\right) \sim \\
& \quad \sim \sum_{l=0}^{n-2}(l+1) \cdots(l+2-n)\left[\frac{1}{l+1}+\cdots+\frac{1}{l+1-n}\right] b_{l} s^{l+1-n}+ \\
& +n ! b_{n-1} \log s+n !(\Psi(n+1)-\Psi(1)) b_{n-1}+ \\
& +\sum_{l=n}^{\infty}\left\{\frac{\Gamma(l+1)}{\Gamma(l+1-n)} \theta\left(x_{0}\right) a_{l} s^{l-n}+\frac{\Gamma(l+2)}{\Gamma(l+2-n)} b_{l}[\Psi(l+2)-\right. \\
& -\Psi(l+2-n)+\log s] s^{l+1-n}
\end{aligned}
$$

where $\Psi(z)=\Gamma^{\prime}(z) / \Gamma(z)$. 
The infinite part of (4.4) as $s \rightarrow 0$ has the correct structure (4.2) and the $b_{\nu}$ are proportional to the $c_{\nu}$ and so are localized at the origin. The expansion of $\theta\left(-x_{0}\right) \delta\left(x^{2}-s\right)$ is obtained from (4.3) by an antichronous Lorentz transformation since $\theta\left(-x_{0}\right) \delta=\overline{\theta\left(+x_{0}\right) \delta}$ :

$$
\begin{aligned}
\theta\left(-x_{0}\right) \delta\left(x^{2}-s\right) & \sim \theta\left(-x_{0}\right) a_{0}(x)+ \\
& +\sum_{n=1}^{\infty}\left[\theta\left(-x_{0}\right) a_{n}(x) s^{n}+b_{n-1}(x) s^{n} \log s\right]
\end{aligned}
$$

where $\theta\left(-x_{0}\right) a_{n}(x)=\overline{\theta\left(x_{0}\right) a_{n}(x)}$ and $\overline{b_{n}(x)}=b_{n}(x)$.

By means of (4.3) and (4.5) we get

$$
\varepsilon\left(x_{0}\right) \delta\left(x^{2}-s\right) \sim \sum_{n=0}^{\infty} \varepsilon\left(x_{0}\right) a_{n}(x) s^{n} .
$$

For $s<0$ we start from the integral

$\delta^{(n)}\left(x^{2}-s\right)\langle\varphi(x)\rangle=2 \pi(-1)^{n} \int_{-\infty}^{\infty} \frac{d^{n}}{d s^{n}}\left[\Phi\left(x_{0}, x_{0}^{2}-s\right) \sqrt{\left.x_{0}^{2}-s\right]} d x_{0}\right.$

and arrive at

$$
\delta\left(x^{2}-s\right) \sim a_{0}(x)+\sum_{n=1}^{\infty} a_{n}(x) s^{n}+2 b_{n-1}(x) s^{n} \log |s|
$$

where $a_{n}(x)=\theta\left(x_{0}\right) a_{n}(x)+\theta\left(-x_{0}\right) a_{n}(x)$.

To determine the distributions $a_{n}(x)$ and $b_{n}(x)$ we proceed as follows: Applying to (4.3) on the one hand the operator $\square$, on the other hand the operator $D_{s}^{+}$and comparing the results we get

$$
\begin{aligned}
& \square\left\{\theta\left(x_{0}\right) a_{n}(x)\right\}=4(n+1) n a_{n+1}+4(2 n+1) b_{n} \\
& \square b_{n}(x)=4(n+1)(n+2) b_{n+1} .
\end{aligned}
$$

From (4.4) and (4.2) it follows that $b_{0}(x)=\pi \delta(x) / 2$ and with (4.9) we obtain

$$
b_{n}(x)=\pi \square^{n} \delta(x)\left[2^{2 n+1} n !(n+1) !\right]^{-1} .
$$

Since $\theta\left(x_{0}\right) \delta\left(x^{2}-s\right)$ is infinitely differentiable in $R^{4}-\mathbf{0}$ at $s=0$ we have in $R^{4}-\mathbf{0}$ the asymptotic Taylor expansion

$$
\theta\left(x_{0}\right) \delta\left(x^{2}-s\right) \sim \sum_{n=0}^{\infty}(-1)^{n} \theta\left(x_{0}\right) \delta^{(n)}\left(x^{2}\right) s^{n} / n ! .
$$

Comparing this result with (4.3) we find $\theta a_{n}(x)=(-1)^{n} \theta \delta^{(n)}\left(x^{2}\right) / n$ ! in $R^{4}-\mathbf{0}$. Since (as the coefficient of an asymptotic expansion whose existence has been proved) $\theta\left(x_{0}\right) a_{n}(x)$ is a well defined distribution in $R^{4}$ we define in $R^{4}$

$$
\mathbf{E}\left\{\theta\left(x_{0}\right) \delta^{(n)}\left(x^{2}\right)\right\}:=(-1)^{n} n ! \theta\left(x_{0}\right) a_{n}(x) .
$$


In order to construct an $a$-dependent extension according to (3.1) we perform a dilatation in (4.3). Noting that $\theta \delta\left(a^{2} x^{2}-s\right)=a^{-2} \theta \delta\left(x^{2}-s / a^{2}\right)$ for $s>0$ and comparing the coefficients of the respective asymptotic expansions one finds

$$
\mathbf{U}_{a}\left\{\theta\left(x_{0}\right) a_{n}(x)\right\}=\left(a^{2}\right)^{-n-1}\left[\theta\left(x_{0}\right) a_{n}(x)-\log a^{2} b_{n-1}(x)\right] .
$$

Since in $R^{4}-\mathbf{0}$

$$
\mathbf{U}_{a}\left\{\theta\left(x_{0}\right) \delta^{(n)}\left(x^{2}\right)\right\}=\left(a^{2}\right)^{-n-1} \theta\left(x_{0}\right) \delta^{(n)}\left(x^{2}\right),
$$

we obtain in virtue of $(3.1)$

$$
\begin{aligned}
\mathbf{E}_{a}\left\{\theta\left(x_{0}\right) \delta^{(n)}\left(x^{2}\right)\right\} & =: \theta\left(x_{0}\right) \delta_{a}^{(n)}\left(x^{2}\right) \\
& =\theta\left(x_{0}\right) \delta_{a=1}^{(n)}\left(x^{2}\right)+\frac{(-1)^{n} \pi \log a^{2}}{2^{2 n-1}(n-1) !} \square^{n-1} \delta(x) .
\end{aligned}
$$

With this explicit $a$-dependence at hand the behaviour under a change of scale follows according to $(3.3)$ :

$$
\begin{aligned}
& \mathbf{U}_{a^{\prime}}\left\{\theta\left(x_{0}\right) \delta_{a}^{(n)}\left(x^{2}\right)\right\}=\left(a^{2}\right)^{-n-1} \theta\left(x_{0}\right) \delta_{a / a^{\prime}}^{(n)}\left(x^{2}\right) \\
= & \left(a^{2}\right)^{-n-1}\left[\theta\left(x_{0}\right) \delta_{a}^{(n)}\left(x^{2}\right)-\frac{(-1)^{n} \pi \log a^{\prime 2}}{2^{2 n-1}(n-1) !} \square^{n-1} \delta(x)\right] .
\end{aligned}
$$

We see that $\theta\left(x_{0}\right) \delta_{a}^{(n)}\left(x^{2}\right)$ is pseudo-homogeneous.

Finally we write (4.3) in terms of $\theta\left(x_{0}\right) \delta_{a}^{(n)}\left(x^{2}\right)$ and $\square^{n} \delta(x)$, viz.

$$
\begin{aligned}
& \theta\left(x_{0}\right) \delta\left(x^{2}-s\right) \sim \theta\left(x_{0}\right) \delta\left(x^{2}\right)+ \\
& +\sum_{n=1}^{\infty}\left[\frac{(-1)^{n}}{n !} \theta\left(x_{0}\right) \delta_{a}^{(n)}\left(x^{2}\right) s^{n}+\frac{\pi \log \left(s / a^{2}\right) \square^{n-1} \delta(x)}{2^{2 n-1} n !(n-1) !}\right] .
\end{aligned}
$$

The corresponding formulas for $\theta\left(-x_{0}\right) \delta_{a}^{(n)}\left(x^{2}\right)$ and $\delta_{a}^{(n)}\left(x^{2}\right)$ are obtained in a similar way. $\varepsilon\left(x_{0}\right) \delta^{(n)}\left(x^{2}\right)=\theta\left(x_{0}\right) \delta_{a}^{(n)}+\theta\left(-x_{0}\right) \delta_{a}^{(n)}$ is of course independent of $a$ and homogeneous.

We are now able to establish the connection of our general delta function with the corresponding distributions of METHÉE and GeLFAND et al. [2], [5].

Methée has chosen the finite part of (4.4) as an extension of $\theta\left(x_{0}\right) \delta^{(n)}\left(x^{2}\right)$ to $R^{4}$. That is to say, as $s$ tends to zero we have

$$
\begin{aligned}
\operatorname{Pf}\left\{\theta\left(x_{0}\right) \delta^{(n)}\left(x^{2}\right)\right\} & =(-1)^{n} n !\left[a_{n}+(\Psi(n+1)-\Psi(1)) b_{n-1}\right] \\
& =\left[\theta\left(x_{0}\right) \delta_{a}^{(n)}\left(x^{2}\right)\right]_{a^{2}}=\exp (\Psi(n+1)-\Psi(1)) .
\end{aligned}
$$

GELFAND et al. introduced two distributions $\delta_{1,2}^{(n)}\left(x^{2}\right)$ which in terms of our residue formula may be written as follows

$$
\begin{aligned}
& \delta_{1}^{(n)}\left(x^{2}\right)\langle\varphi(x)\rangle=\operatorname{Res}_{z=0}\left\{\frac{2 \pi}{z} \int_{0}^{\infty} \tau^{-n-z}\left[\frac{d^{n}}{d \omega^{n}}\left(\omega^{1 / 2} \hat{\Phi}(\tau, \omega \tau)\right)\right]_{\omega=1} d \tau\right\} \\
& \delta_{2}^{(n)}\left(x^{2}\right)\langle\varphi(x)\rangle=\operatorname{Res}_{z=0}\left\{\frac{2 \pi}{z} \int_{0}^{\infty} \varrho^{-n-z}\left[\frac{d^{n}}{d \omega^{n}}\left(\omega^{-1 / 2} \hat{\Phi}(\omega \varrho, \varrho)\right)\right]_{\omega=1} d \varrho\right\}
\end{aligned}
$$


where $\hat{\Phi}(\tau, \varrho)=\frac{1}{2}\left(\Phi\left(\tau^{1 / 2}, \varrho\right)+\Phi\left(-\tau^{1 / 2}, \varrho\right)\right)($ see $(4.1)), \tau=x_{0}^{2}$ and $\varrho=\mathrm{x}^{2}$. With these definitions it follows (cf. [5]) that

$$
\operatorname{Res}_{\lambda=-n-1}\left\{\theta\left(x^{2}\right)\left(x^{2}\right)^{\lambda}\right\}=\frac{(-1)^{n}}{n !} \delta_{1}^{(n)}\left(x^{2}\right)+\frac{\pi[\Psi(1 / 2)-\Psi(2)]}{2^{2 n-2} n(n-1) !} \square^{n-1} \delta(x)
$$

and

$$
\delta_{1}^{(n)}\left(x^{2}\right)-\delta_{2}^{(n)}\left(x^{2}\right)=\frac{(-1)^{n} 2 \pi}{2^{2 n-2}(n-1) !} \square^{n-1} \delta(x) .
$$

We shall show in Sec. 6 that

$$
\operatorname{Res}_{\lambda=-n-1}\left\{\theta\left(x^{2}\right)\left(x^{2}\right)^{\lambda}\right\}=\left[\frac{(-1)^{n}}{n !} \delta_{a}^{(n)}\left(x^{2}\right)\right]_{a=1} .
$$

Since $\operatorname{Res} \theta\left(x^{2}\right)\left(x^{2}\right)^{\lambda}$ is well defined we obtain

$$
\delta_{1,2}^{(n)}\left(x^{2}\right)=\left[\delta_{a}^{(n)}\left(x^{2}\right)\right]_{a^{2}}=\exp ( \pm 1-2 \log 2) .
$$

Formula (3.4) permits us to calculate the $a$-dependence of $\operatorname{Pf}\left\{\delta^{(n)}\left(x^{2}\right)\right\}$ and $\delta_{1 / 2}^{(n)}\left(x^{2}\right)$ and to discuss their behaviour under a dilatation.

Some formulas are yet of interest. From (4.9) we find at once

$$
\square\left\{\theta\left(x_{0}\right) \delta_{a}^{(n)}\left(x^{2}\right)\right\}=-4 n \delta_{a}^{(n+1)}\left(x^{2}\right)+\frac{\pi(-1)^{n}}{2^{2 n-1}(n+1) !} \square^{n} \delta(x) .
$$

Applying the chain rule to (4.3) we obtain

$$
\frac{\partial}{\partial x_{v}} \delta_{a}^{(n)}\left(x^{2}\right)=2 g_{v v} x_{\nu} \delta_{a}^{(n+1)}\left(x^{2}\right)-\frac{4 g_{v v} \pi(-1)^{n} x_{v}}{2^{2 n+1}(n+1) !} \square^{n} \delta(x)
$$

where $g_{00}=-g_{i i}=1$. One notices that, once the origin of the light cone is included, the chain rule is no longer valid. Taking account of $\left(x^{2}\right)^{l} \delta\left(x^{2}-s\right)=s^{l} \delta\left(x^{2}-s\right)$ for $s \neq 0$ it follows from (4.3) that

$n, m=0,1,2, \ldots$ and

$$
\left(x^{2}\right)^{m} \square^{n} \delta(x)=\frac{4^{n} n !(n+1) !}{(n-m) !(n-m+1) !} \square^{n-m} \delta(x),
$$

$$
\left(x^{2}\right)^{m} \delta_{a}^{(n)}\left(x^{2}\right)=\frac{(-1) n^{m} !}{(n-m) !} \delta_{a}^{(n-m)}\left(x^{2}\right) .
$$

Since in the corresponding formulas for $\varepsilon\left(x_{0}\right) \delta^{(n)}\left(x^{2}\right)$ the terms located at the origin cancel each other, the same rules hold for this distribution as if $x^{2}=0$ were a regular surface.

\section{Asymptotic and Laurent Expansions, Equivalent Extensions}

Let $f(s)$ be locally summable for $s>0$ with an algebraic singularity at $s=0$. We assume for simplicity that $f(s)$ admits an asymptotic expansion in $s^{\mu} \log s^{v}$ of infinite order at $s=0$. (For the following it would be sufficient to require that $f(s)$ has an infinite part, i.e., admits an asymptotic expansion up to order $0(1)$.) Hence, as $s \rightarrow 0$

$$
f(s) \theta\left(x_{0}\right) \delta\left(x^{2}-s\right) \sim \sum_{l=1}^{\infty} \sum_{m=1}^{n_{l}} \theta\left(x_{0}\right) a_{l m}(x) s^{x_{l}-1} \log ^{m-1} s
$$


with $\operatorname{Re} x_{l}<\operatorname{Re} x_{l+1}$. The $\theta\left(x_{0}\right) a_{l m}$ are finite linear combinations of the $\theta\left(x_{0}\right) a_{n}$ and $b_{n}$ of (4.3) and therefore localized on the forward cone $\left\{x: x^{2}=0, x_{0} \geqq 0\right\}$. Integrating (5.1) we get

$$
\begin{aligned}
\int_{t}^{\infty} s^{-z} f(s) \theta\left(x_{0}\right) & \delta\left(x^{2}-s\right) d s \sim \\
\sim & -\sum_{l, m=1}^{\infty, n_{l}}\left[\theta\left(x_{0}\right) a_{l m}(x) F\left(x_{l}-z, m, t\right)\right]+\theta\left(x_{0}\right) K(x, z)
\end{aligned}
$$

where

$$
\begin{aligned}
& F\left(\varkappa_{l}-z, m, t\right)=\int^{t} s^{\varkappa_{l}-1-z} \log ^{m-1} s d s \\
& = \begin{cases}\frac{(-1)^{m-1}(m-1) !}{\left(\varkappa_{l}-z\right)^{m}} t^{\varkappa_{l}-z} \sum_{\mu=0}^{m-1} \frac{(-1)^{m-1-\mu}}{(m-1-\mu) !}\left(\varkappa_{l}-z\right)^{m-1-\mu} \log ^{m-1-\mu} t ; \\
\left(\log ^{m} t\right) / m & x_{l}-z \neq 0 \\
& ; x-z=0\end{cases}
\end{aligned}
$$

and $\theta\left(x_{0}\right) K(x ; z)$ is a distribution independent of $t$. Observe that the $F\left(\varkappa_{l}-z, m, t\right)$ either vanish or tend to infinity as $t \rightarrow 0$. Let us choose, for a fixed but arbitrary $z=z_{0}$, an integer $L=L\left(z_{0}\right)$ such that all $F\left(x_{l}-z, m, t\right)$ with $l>L$ vanish as $t \rightarrow 0$. Then we have

$$
\begin{aligned}
& \theta\left(x_{0}\right) K\left(x ; z_{0}\right)=\operatorname{Pf} \int_{t}^{\infty} s^{-z_{0}} f(s) \theta\left(x_{0}\right) \delta\left(x^{2}-s\right) \\
& =\lim _{t \rightarrow 0}\left[\int_{0}^{\infty} d s s^{-z_{0}} f(s) \theta\left(x_{0}\right) \delta\left(x^{2}-z\right)+\sum_{l=1}^{L} \sum_{m=1}^{n_{l}} \theta\left(x_{0}\right) a_{l m}(x) F\left(x_{l}-z, m, t\right)\right] .
\end{aligned}
$$

The same $L$ works for all $z$ with $\operatorname{Re} z<\operatorname{Re} z_{0}$. For $t>0$ the expression in [...], (5.4), is analytic for all $z$ with $\operatorname{Re} z<\operatorname{Re} z_{0}$ and $z \neq \varkappa_{l}$. Since in (5.4) $z_{0}$ is arbitrary and we have uniform convergence in all bounded domains of the $z$-plane, $\theta\left(x_{0}\right) K(x ; z)$ is analytic for all $z \neq \varkappa_{l}$. Especially for $z=0$ we obtain, if no $x_{l}$ is equal to zero,

$$
\begin{aligned}
\theta\left(x_{0}\right) K(x ; 0) & =\operatorname{Pf}_{t \rightarrow 0} \int_{t}^{\infty} f(s) \theta\left(x_{0}\right) \delta\left(x^{2}-s\right) d s \\
& =\underset{z=0}{\operatorname{A.C}} \int_{0}^{\infty} s^{-z} f(s) \theta\left(x_{0}\right) \delta\left(x^{2}-s\right) d s .
\end{aligned}
$$

A.C. means analytic continuation from the region $\operatorname{Re}(z)$ large negative such that the series in $(5.4)-$ the infinite part - vanishes at $t=0$. Let now $\varkappa_{k}$ be equal to zero for a fixed $k$. Then we get from (5.3)

$$
\begin{aligned}
F(-z, m, t) & =(-1)(m-1) ! z^{-m}+\left(\log ^{m} t\right) / m+0(z) \\
& =-(m-1) ! z^{-m}+F(0, m, t)+0(z) .
\end{aligned}
$$


Substituting (5.6) into (5.4), shifting all pole terms of $F\left(x_{l}-z, m, t\right)$ with $\varkappa_{k}=0$ to the left hand side and passing to the limit $z=0$, yields

$$
\begin{aligned}
& \lim _{z \rightarrow 0}\left[\text { A.C. } \int_{0}^{\infty} s^{-z} f(s) \theta\left(x_{0}\right) \delta\left(x^{2}-s\right) d s+\sum_{m=1}^{n_{k}} \theta\left(x_{0}\right) a_{k m}(x)(m-1) ! z^{-m}\right] \\
& =\lim _{t \rightarrow 0}\left[\int_{t}^{\infty} f(s) \theta\left(x_{0}\right) \delta\left(x^{2}-s\right) d s+\sum_{l=1}^{L} \sum_{m=1}^{n_{k}} \theta\left(x_{0}\right) a_{l m}(x) F\left(x_{l}, m, t\right)\right] .
\end{aligned}
$$

The existence of the right hand side implies that of the left hand side. We summarize (5.5) and (5.7) into the statement

$$
\operatorname{Res}_{z=0}\left[\frac{1}{z} \int_{0}^{\infty} f(s) s^{-z} \theta\left(x_{0}\right) \delta\left(x^{2}-s\right) d s\right]=\operatorname{Pf}_{t \rightarrow 0} \int_{t}^{\infty} f(s) \theta\left(x_{0}\right) \delta\left(x^{2}-s\right) d s .
$$

Since this equality holds for $a=1$ we infer from (3.4) its validity for all $a>0$. Hence

$\operatorname{Res}_{z=0}\left[\frac{1}{z} \int_{0}^{\infty} f(s)\left(\frac{a^{2}}{s}\right)^{z} \theta\left(x_{0}\right) \delta\left(x^{2}-s\right) d s\right]=\operatorname{Pf}_{t \rightarrow 0} \int_{a^{2} t}^{\infty} f(s) \theta\left(x_{0}\right) \delta\left(x^{2}-s\right) d s .(5.8)$

Henceforth we denote the extensions (5.8) by $\theta\left(x_{0}\right) f_{a}\left(x^{2}\right)$ or by $\left[\theta\left(x_{0}\right) f\left(x^{2}\right)\right]_{a}$. This explicit calculation shows that $\theta f_{a}$ is not constant in $a$ if and only if in (5.1) one of the $\varkappa_{l}$ is equal to zero, i.e., if $\operatorname{Pif}\{f(s)\}$ contains one or more terms of the form $s^{-n} \log ^{m} s, n=1,2, \ldots$, $m=0,1,2, \ldots$.

From (5.7) we see that

$$
\text { A.C. } \int_{0}^{\infty} s^{-z} f(s) \theta\left(x_{0}\right) \delta\left(x^{2}-s\right) d s+\sum_{m=1}^{n_{k}} \theta\left(x_{0}\right) a_{k m}(x)(m-1) ! z^{-m}
$$

is analytic in a neighbourhood of $z=0$ and thus has a Taylor expansion. The $n$-th derivative is

$$
\begin{aligned}
(-1)^{n}\left[\text { A.C. } \int_{0}^{\infty} s^{-z} \log ^{n} s f(s) \theta\left(x_{0}\right) \delta\left(x^{2}-s\right) d s+\right. & \\
& \left.+\sum_{m=0}^{n_{k}} \theta\left(x_{0}\right) a_{k m}(m+n-1) ! z^{-m-n}\right]
\end{aligned}
$$

whose value at $z=0$ is just $\left[\theta\left(x_{0}\right)\left(\log ^{n} x^{2}\right) f\left(x^{2}\right)\right]_{a=1}$. We therefore obtain the following Laurent expansion at $z=0$,

$$
\begin{aligned}
& {\left[\theta\left(x_{0}\right)\left(x^{2}\right)^{-z} f\left(x^{2}\right)\right]_{a}} \\
& =-\sum_{m=1}^{n_{k}}(m-1) ! \theta\left(x_{0}\right) a_{k m}(x) z^{-m}+\left[\theta\left(x_{0}\right) f\left(x^{2}\right)\right]_{a=1}+ \\
& +\sum_{n=1}^{\infty} \frac{(-z)^{n}}{n !}\left[\theta\left(x_{0}\right)\left(\log ^{n} x^{2}\right) f\left(x^{2}\right)\right]_{a=1} .
\end{aligned}
$$


The rhs of this expansion does of course not depend on $a$ since in a neighbourhood of $z=0(z \neq 0)\left[\theta\left(x_{0}\right)\left(x^{2}\right)^{-z} f\left(x^{2}\right)\right]_{a}$ is analytic in $z$ and does not depend on $a$. Multiplying (5.9) with the Taylor series of $\left(a^{2}\right)^{z}$ and collecting the $z$-free terms yields

$$
\left[\theta\left(x_{0}\right) f\left(x^{2}\right)\right]_{a}=\left[\theta\left(x_{0}\right) f\left(x^{2}\right)\right]_{a=1}-\sum_{m=1}^{n_{k}}\left(\log ^{m} a^{2}\right) \theta\left(x_{0}\right) a_{k m}(x) / m .
$$

The preceding considerations also demonstrate the existence of A.C. $\int_{0}^{\infty} s^{-z} f(s) \theta\left(x_{0}\right) \delta\left(x^{2}-s\right) d s$ with the exception of isolated poles under the assumption that $f(s)$ possesses a well defined infinite part. In fact, $\underset{t \rightarrow 0}{\operatorname{Pf}} \int_{t}^{\infty} f(s) s^{-z} \theta\left(x_{0}\right) \delta\left(x^{2}-s\right) d s$ generates a construction of this analytic continuation.

The above results are easily extended to $\theta\left(-x_{0}, f_{a}\left(x^{2}\right)\right.$ and $\theta\left(-x^{2}\right) f_{a}\left(x^{2}\right)$.

\section{Distributions of Power and Logarithmic Type}

\subsection{The Distributions $\left[\left(\log ^{m}\left|x^{2}\right|\right)\left(x^{2}\right)^{\lambda}\right]_{a}$}

According to Sec. 4 we have the asymptotic expansions

$$
\begin{aligned}
& s^{\lambda} \log ^{m} s \theta\left(x_{0}\right) \delta\left(x^{2}-s\right) \underset{s \rightarrow+0}{\sim} \sum_{n=1}^{\infty}\left[\theta\left(x_{0}\right) a_{n-1} s^{\lambda+n-1} \log ^{m} s+\right. \\
& \left.+b_{n-2} s^{\lambda+n-1} \log ^{m+1} s\right] \\
& s^{\lambda}\left(\log ^{m}|s|\right) \delta\left(x^{2}-s\right) \underset{s \rightarrow \pm 0}{\sim} \sum_{n=1}^{\infty}(-1)^{n-1}\left[a_{n-1} s^{\lambda+n-1} \log ^{m}|s|+\right. \\
& \left.+2 b_{n-2} s^{\lambda+n-1} \log ^{m+1}|s|\right] \text {. }
\end{aligned}
$$

where $b_{-1}(x):=0$.

They have, by means of the results of Sec. 5, the following statements and formulas as consequences:

The distributions $\left[\theta \log ^{m}\left|x^{2}\right|\left(x^{2}\right)^{\lambda}\right]_{a}\left(\theta\right.$ being either $\theta\left( \pm x_{0}\right)$ or $\left.\theta\left(-x^{2}\right)\right)$ are analytic in the whole $\lambda$-plane except for the points $\lambda=-1,-2, \ldots$ where poles arise. For later purposes we need the following Laurent expansions explicitly

$$
\begin{aligned}
& {\left[\theta\left(x_{0}\right)\left(x^{2}\right)^{-n-z}\right]_{a}=-b_{n-2} z^{-2}-\theta\left(x_{0}\right) a_{n-1} z^{-1}+} \\
& +\left[\theta\left(x_{0}\right)\left(x^{2}\right)^{-n}\right]_{a-1}+\sum_{m=1}^{\infty}\left[\theta\left(x_{0}\right)\left(\log ^{m} x^{2}\right)\left(x^{2}\right)^{-n}\right]_{a=1}(-z)^{m} / m ! \\
& {\left[\varepsilon\left(x_{0}\right)\left(x^{2}\right)^{-n-z}\right]_{a}=-\varepsilon\left(x_{0}\right) a_{n-1} z^{-1}+\left[\varepsilon\left(x_{0}\right)\left(x^{2}\right)^{-n}\right]_{a=1}+0(z)} \\
& {\left[\theta\left(-x^{2}\right)\left|x^{2}\right|^{-n-z}\right]_{a}=(-1)^{n} 2 b_{n-2} z^{-2}+} \\
& +(-1)^{n} a_{n-1} z^{-1}+\left[\theta\left(-x^{2}\right)\left|x^{2}\right|^{-n}\right]_{a=1}+0(z) .
\end{aligned}
$$


From (6.3) we obtain

$$
\begin{aligned}
{\left[\theta\left(x_{0}\right)\left(x^{2}\right)^{-n}\right]_{a}=\left[\theta\left(x_{0}\right)\left(x^{2}\right)^{-n}\right]_{a} } & =1-\log a^{2} \frac{(-1)^{n-1}}{(n-1) !} \delta_{a=1}^{(n-1)}\left(x^{2}\right)- \\
& -\frac{\pi \log ^{2} a^{2}}{2^{2 n-2}(n-1) !(n-2) !} \square^{n-2} \delta(x)
\end{aligned}
$$

and from (6.5) we get

$$
\begin{aligned}
{\left[\theta\left(-x^{2}\right)\left|x^{2}\right|-n\right]_{a}=} & {\left[\theta\left(-x^{2}\right)\left|x^{2}\right|^{-n}\right]_{a=1}+(-1)^{n}\left(\log a^{2}\right) a_{n-1}(x) } \\
& +(-1)^{n}\left(\log ^{2} a^{2}\right) b_{n-2}(x) .
\end{aligned}
$$

Let us define the distribution $\left[\left(x^{2}\right)^{\lambda}\right]_{a}$ by

$$
\left[\left(x^{2}\right)^{\lambda}\right]_{a}=\operatorname{Res}_{z=0}\left[\frac{1}{z} \int_{0}^{\infty} s^{\lambda}\left(\frac{a^{2}}{s}\right)^{z} \delta\left(x^{2}-s\right) d s+e^{i \pi \lambda} \int_{0}^{\infty} s^{\lambda}\left(\frac{a^{2}}{s}\right)^{z} \delta\left(x^{2}+s\right) d s\right]
$$

and observe that the poles at $\lambda=-1,-2, \ldots$ of the terms in (6.8) cancel each other, such that $\left[\left(x^{2}\right)^{\lambda}\right]_{a}$ is analytic in the whole $\lambda$-plane and independent of $a$. For later reference we need the Laurent expansion

$$
\begin{aligned}
& {\left[\theta\left(x_{0}\right)\left(\log x^{2}\right)\left(x^{2}\right)^{-n-z}\right]_{a}} \\
& \quad=\left[\theta\left(x_{0}\right)\left(\log x^{2}\right)\left(x^{2}\right)^{-n}\right]_{a=1}-2 b_{n-2} z^{-3}-\theta\left(x_{0}\right) a_{n-1} z^{-2}+0(z) .
\end{aligned}
$$

Let us consider (6.6) in more detail: apply (3.3) to it and calculate

$$
\mathbf{U}_{a^{\prime}}\left[\theta\left(x_{0}\right)\left(x^{2}\right)^{-n}\right]_{a}=\left(a^{\prime 2}\right)^{-n}\left[\theta\left(x_{0}\right)\left(x^{2}\right)^{-n}\right]_{a / a^{\prime}} .
$$

This distribution is seen to be pseudo-homogeneous (and, indeed is an associated generalized function of second order). On the subspace of test functions whose meanvalue over the light-cone vanishes sufficiently strongly, this distribution is equivalent to an ordinary homogeneous function. Hence $x$ may be taken to have dimension of length. Then also $a$ must be considered as a length and although all three terms of the $r h s$ of (6.6) appear to have illegitimate dimensional arguments their sum is dimensionally correct. (Things are similar as in $\log ^{2}\left(x^{2} / a^{2}\right)=\log ^{2} x^{2}-$ $-2 \log a^{2} \log x^{2}+\log ^{2} a^{2}$,

which is also an associated function of second order.) This is explicitly seen by calculating the Fourier transform of $\left[\theta\left(x_{0}\right)\left(x^{2}\right)^{-n}\right]_{a}$ (cf. Sec. 7).

\subsection{The Distributions $\left(x^{2} \pm i 0\right)^{\lambda}$ and $\left(x^{2} \pm i x_{0} 0\right)^{\lambda}$}

In the one dimensional case the relation

$$
\begin{aligned}
(s \pm i 0)^{\lambda}: & =\lim _{\varepsilon \rightarrow 0}|s|^{\lambda} \exp [i \arg (s \pm i \varepsilon) \lambda] \\
& =\theta(s) s^{\lambda}+e^{ \pm i \pi \lambda} \theta(-s)|s|^{\lambda}
\end{aligned}
$$


holds. Thus we define

$$
\begin{gathered}
{\left[\left(x^{2} \pm i 0\right)^{\lambda}\right]_{a}=\operatorname{Res}_{z=0}\left[\frac{1}{z} \int_{-\infty}^{\infty}(s \pm i 0)^{\lambda}\left(\frac{a^{2}}{s \pm i 0}\right)^{z} \delta\left(x^{2}-s\right) d s\right]} \\
=\operatorname{Res}_{z=0}\left[\frac{1}{z} \int_{0}^{\infty} s^{\lambda}\left(\frac{a^{2}}{s}\right)^{z}\left\{\delta\left(x^{2}-s\right)+e^{ \pm i \pi(\lambda-z)} \delta\left(x^{2}+s\right)\right\} d s\right] .
\end{gathered}
$$

Note the difference with (6.8). By means of (6.3) and (6.5) we find

$$
\begin{aligned}
{\left[\left(x^{2} \pm i 0\right)^{-n-z}\right]_{a}=} & \mp 2 \pi i b_{n-2} z^{-1}+\left[\left(x^{2} \pm i 0\right)^{-n}\right]_{a=1}+ \\
& +\sum_{m=1}^{\infty}\left[\left(x^{2} \pm i 0\right)^{-n} \log ^{m}\left(x^{2} \pm i 0\right)\right]_{a=1}(-z)^{m} / m !
\end{aligned}
$$

where

$$
\begin{aligned}
{\left[\left(x^{2} \pm i 0\right)^{-n}\right]_{a=1}=\left(x^{2}\right)^{-n} \mp i \pi \frac{(-1)^{n-1}}{(n-1) !} \delta_{a=1}^{(n-1)}\left(x^{2}\right)-} & \\
& -\frac{\pi^{3} \square^{n-2} \delta(x)}{2^{2} \frac{n-2}{(n-1) !(n-2) !}}
\end{aligned}
$$

and

$$
\log \left(x^{2} \pm i 0\right)=\log \left|x^{2}\right| \pm i \pi \theta\left(-x^{2}\right) .
$$

The $a$-dependence turns out to be

$$
\left[\left(x^{2} \pm i 0\right)^{-n}\right]_{a}=\left[\left(x^{2} \pm i 0\right)^{-n}\right]_{a=1} \mp \frac{i \pi^{2} \log a^{2} \square^{n-2} \delta(x)}{2^{2 n-3}(n-1) !(n-2) !} .
$$

From (6.12) and (6.14) we obtain

$$
\left[\left(x^{2}-i 0\right)^{-n}\right]_{a}-\left[\left(x^{2}+i 0\right)^{-n}\right]_{a}=2 \pi i \frac{(-1)^{n-1}}{(n-1) !} \delta_{a}^{(n-1)}\left(x^{2}\right) .
$$

Formula (6.15) also justifies our choice of the extension $\delta_{a}^{(n)}\left(x^{2}\right)$.

To define the Wightman-type distributions $\left(x^{2} \pm i x_{0} 0\right)^{\lambda}$ we note that for $\operatorname{Re} \lambda>0$

$$
\begin{aligned}
& \left(x^{2}-i x_{0} 0\right)^{\lambda}:=\lim _{\varepsilon \rightarrow 0} e^{-i \pi \lambda} \cdot\left(\mathbf{x}^{2}-\left(x_{0}-i \varepsilon\right)^{2}\right)^{\lambda} \\
& \quad=e^{-i \pi \lambda}\left[e^{i \pi \lambda} \theta\left(x_{0}\right)\left(x^{2}\right)^{\lambda}+e^{-i \pi \lambda} \theta\left(-x_{0}\right)\left(x^{2}\right)^{\lambda}+\theta\left(-x^{2}\right)\left|x^{2}\right|^{\lambda}\right] .
\end{aligned}
$$

In (6.16) we have separated the factor $\exp (-i \pi \lambda)$ to make the definition Lorentz invariant. On the other hand we define

$$
\left.\left(x^{2}+i x_{0} 0\right)^{\lambda}:=\overline{\left(x^{2}-i x_{0} 0^{\lambda}\right.}\right) .
$$

In virtue of the formulas of Sec. 6.1 we see that in (6.16) the poles cancel and that $\left(x^{2} \pm i x_{0} 0\right)^{\lambda}$ is analytic in the whole $\lambda$-plane. For $\lambda=-n$ we obtain

$$
\begin{aligned}
\left(x^{2} \pm i x_{0} 0\right)^{-n}=\left(x^{2}\right)^{-n} \mp i \pi \frac{(-1)^{n-1}}{(n-1) !} \varepsilon\left(x_{0}\right) & \delta^{n-1}\left(x^{2}\right)+ \\
& +\frac{\pi^{3} \square^{n-2} \delta(x)}{2^{2 n-3}(n-1) !(n-2) !}
\end{aligned}
$$


and

$$
\left(x^{2}-i x_{0} 0\right)^{-n}-\left(x^{2}+i x_{0} 0\right)^{-n}=2 \pi i \frac{(-1)^{n-1}}{(n-1) !} \varepsilon\left(x_{0}\right) \delta^{(n-1)}\left(x^{2}\right) .
$$

\subsection{Differentiation Formulas}

By means of the formulas of Sec. 3.3 one easily derives the following relations

$$
\square\left[\theta\left(x_{0}\right)\left(x^{2}\right)^{\lambda}\right]_{a}=4 \lambda(\lambda+1)\left[\theta\left(x_{0}\right)\left(x^{2}\right)^{\lambda}\right]_{a}
$$

for $\lambda \neq 0,-1,-2, \ldots$;

$\square\left[\theta\left(x_{0}\right)\left(x^{2}\right)^{-n}\right]_{a}=4 n(n-1)\left[\theta\left(x_{0}\right)\left(x^{2}\right)^{-n-1}\right]_{a}-$

and

$$
-4(2 n-1) \frac{(-1)^{n} \theta\left(x_{0}\right) \delta_{a}^{(n)}\left(x^{2}\right)}{n !}-\frac{4 \pi \square^{n-1} \delta(x)}{2^{2 n-1} n !(n-1) !}
$$

$\square\left[\theta\left(-x^{2}\right)\left|x^{2}\right|^{-n}\right]_{a}=-4 n(n-1)\left[\theta\left(-x^{2}\right)\left|x^{2}\right|^{-n-1}\right]_{a}+$

for $n=2,3,4 \ldots$.

$$
+4(2 n-1) \delta_{a}^{(n)}\left(x^{2}\right) / n !+\frac{(-1)^{n} 8 \pi \square^{n-1} \delta(x)}{2^{2 n-1} n !(n-1) !}
$$

Combining (6.20) with (6.21) we obtain

$$
\square\left(x^{2}\right)^{-n}=4 n(n-1)\left(x^{2}\right)^{-n-1} .
$$

Let us moreover compile the following formulas

$$
\begin{gathered}
\square \theta\left(x_{0}\right)=4 \theta\left(x_{0}\right) \delta\left(x^{2}\right) \\
\square \theta\left(-x^{2}\right)=-4 \delta\left(x^{2}\right) \\
\square\left[\theta\left(x_{0}\right)\left(x^{2}\right)^{-1}\right]_{a}=4 \delta_{a}^{\prime}\left(x^{2}\right)-2 \pi \delta(x) \\
\square\left[\theta\left(-x^{2}\right)\left(x^{2}\right)^{-1}\right]_{a}=-4 \delta_{a}^{\prime}\left(x^{2}\right)+4 \pi \delta(x) \\
\square\left[\theta\left(x_{0}\right) \log x^{2}\right]=4 \theta\left(x_{0}\right)\left(x^{2}\right)^{-1}+4 \theta\left(x_{0}\right) \delta\left(x^{2}\right) \\
\square\left[\left(x^{2} \pm i 0\right)^{-n}\right]_{a}=4 n(n-1)\left[\left(x^{2} \pm i 0\right)^{-n-1}\right]_{a} \mp \\
\mp \frac{8(2 n-1) i \pi^{2} \square^{n-1} \delta(x)}{2^{2 n-1} n !(n-1) !} .
\end{gathered}
$$

Finally we discuss, guided by an example, the $a$-dependence of singular distributions generated by differentiating regular ones. For all $x$ with $x^{2} \neq 0$ we have

$$
\left(x^{2}\right)^{-n}=-\square^{n}\left[\log ^{2}\left(x^{2}\right)-2 \log x^{2}+2\right] /\left(2 \cdot 4^{n}(n-1) !(n-2) !\right) .
$$

Thus we define

$$
\begin{aligned}
& \widehat{\mathbf{E}}\left\{\theta\left(x_{0}\right)\left(x^{2}\right)^{-n}\right\} \\
& \quad:=-\square^{n}\left\{\theta\left(x_{0}\right)\left[\log ^{2}\left(x^{2}\right)-2 \log x^{2}+2\right]\right\} /\left(2 \cdot 4^{n}(n-1) !(n-2) !\right),
\end{aligned}
$$


and the $a$-dependent distribution is according to (3.1) given by $\widehat{\mathbf{E}}_{a}\left\{\theta\left(x_{0}\right)\left(x^{2}\right)^{-n}\right\}$

$=\frac{-1}{2 \cdot 4^{n}(n-1) !(n-2) !} \square^{n}\left\{\theta\left(x_{0}\right)\left[\left(\log \left(x^{2} / a^{2}\right)\right)^{2}-2\left(\log \left(x^{2} / a^{2}\right)\right)+2\right]\right\}$.

For $n=2$ we find

$$
\hat{\mathbf{E}}_{a}\left\{\theta\left(x_{0}\right)\left(x^{2}\right)^{-2}\right\}=\left[\theta\left(x_{0}\right)\left(x^{2}\right)^{-2}\right]_{a}+\theta\left(x_{0}\right) \delta_{a}^{\prime}\left(x^{2}\right) .
$$

Since in (6.31) the $r h s$ is dimensionally correct we have constructed another dimensionally correct extension of the function $\theta\left(x_{0}\right)\left(x^{2}\right)^{-2}$. Formula (6.30) shows that the $a$-dependence is due to the sharp boundary in $x$-space $\left(\theta\left(x_{0}\right)\right.$ and singularity). The distribution defined by $(6.29)$ in terms of generalized derivatives is independent of $a$ because of the absence of the $\theta$-function although $\int_{R^{4}}\left(x^{2}\right)^{-n} \varphi(x) d x$ exhibits logarithmic divergencies.

\section{Fourier Transforms in Terms of Spectral Representations}

\section{\%.1. Definitions}

For $\varphi(x) \in \mathscr{D}\left(R^{4}\right)$ we define

$$
\psi(y):=\int_{R^{4}} \varphi(x) e^{i x y} d x=F \varphi(x)
$$

where $y=y^{\prime}+i y^{\prime \prime}$ is a complex four vector, $x y=x_{0} y_{0}-\mathbf{x} \mathbf{y}$. The set of all functions $\psi(y)$ forms the space $\mathscr{Z}\left(C^{4}\right)$, i.e.,

$$
\left|y_{v}^{k} \psi(y)\right| \leqq C_{k} \exp \left(b\left|y_{v}^{\prime \prime}\right|\right)
$$

for all $k=0,1,2, \ldots$ and $\nu=0,1,2,3 . C_{k}$ and $b$ are constants depending on $\psi$. The subspace of $\mathscr{Z}\left(C^{4}\right)$ defined by those $\psi$ which are the Fourier transforms of $\varphi$ 's from $D\left(R^{4}-\mathbf{0}\right)$ is denoted by $\mathscr{Z}_{0}\left(C^{4}\right)$. If $\psi(y) \in \mathscr{Z}_{0}\left(R^{4}\right)$ there holds

for all $l=0,1,2, \ldots$.

$$
\int_{R^{4}}\left(y^{2}\right)^{l} \psi(y) d y=0
$$

Let $f(x) \in \mathscr{D}^{\prime}\left(R^{4}\right)$. We define

$(F f)(y)\langle\psi(y)\rangle \equiv \tilde{f}(y)\langle\psi(y)\rangle$

$$
:=f(x)\langle F \psi(y)\rangle=(2 \pi)^{-4} f(x)\langle\varphi(-x)\rangle .
$$

The mapping $\mathscr{D}^{\prime}\left(R^{4}\right)$ onto $\mathscr{Z}^{\prime}\left(C^{4}\right)$ via the Fourier transform $F$ has a unique inverse. Since a Lorentz transformation commutes with the Fourier transform we have the

Statement. $\tilde{f}(y) \in \mathscr{Z}^{\prime}\left(C^{4}\right)$ is invariant under a real Lorentz transformation if and only if $f(x)$ is invariant under this transformation.

Since in what follows we are only concerned with invariant distributions, all formulas remain valid for the Euclidean Fourier transformation.

Commun. math. Phys., Vol. 7 
We note that

and

$$
F \bar{f}=\overline{F f}
$$

$$
F \mathbf{U}_{a} f=a^{-4} \mathbf{U}_{1 / a} F f .
$$

Let $f$ be pseudo-homogeneous of degree $\lambda$. Setting $F f_{a=1}=\tilde{f}_{a=1}$ we find

$$
F f_{a}=a^{\lambda+4} \mathbf{U}_{a} \tilde{f}_{a=1}
$$

\subsection{Fourier Transforms of Delta Functions}

As is well known (cf., Ref. [11]), there hold the following relations.

$$
\begin{gathered}
F \delta\left(x^{2}-s\right)=(2 \pi)^{3} \Delta^{(1)}(y, s) \equiv 2 \pi^{2} s \operatorname{Im}\left\{H_{1}^{(1)}\left(\sqrt{s y^{2}}\right) / \sqrt{s y^{2}}\right\}, s \neq 0, \\
F \delta\left(x^{2}\right)=-4 \pi\left(y^{2}\right)^{-1} \\
F \varepsilon\left(x_{0}\right) \delta\left(x^{2}-s\right)=-i(2 \pi)^{3} \Delta(y, s) \\
=4 \pi^{2} i \varepsilon\left(y_{0}\right) \delta\left(y^{2}\right)-2 \pi^{2} i s \varepsilon\left(y_{0}\right) \mathscr{J}_{1}\left(\sqrt{s y^{2}}\right) / \sqrt{s y^{2}}, s>0 \\
F \varepsilon\left(x_{0}\right) \delta\left(x^{2}\right)=4 \pi^{2} i \varepsilon\left(y_{0}\right) \delta\left(y^{2}\right) \\
F \theta\left(x_{0}\right) \delta\left(x^{2}-s\right)=i(2 \pi)^{3} \Delta^{+}(-y, s) \\
\equiv-i \pi^{2} s\left[H_{1}^{(1)}\left(\sqrt{s \zeta^{2}}\right) / \sqrt{s \zeta^{2}}\right]_{\zeta^{2}}=y^{2}+i y_{0} 0
\end{gathered}
$$

By means of the series expansion of the Hankel function we obtain from (7.7)

$F \delta\left(x^{2}-s\right)=-\frac{4 \pi}{y^{2}}-$

$-\sum_{n=1}^{\infty} \frac{(-1)^{n} \pi\left(y^{2}\right)^{n-1} s^{n}}{4^{n-1} n !(n-1) !}\left[\log \left|\frac{y^{2} a^{2}}{4}\right|-\Psi(n)-\Psi(n+1)+\log \frac{s}{a^{2}}\right]$.

On the other hand we know from $\left(4.3^{\prime}\right)$ that

$F \delta\left(x^{2}-s\right) \sim F \delta\left(x^{2}\right)+$

$$
+\sum_{n=1}^{\infty}\left[\frac{(-1)^{n}}{n !} s^{n} F \delta_{a}^{(n)}\left(x^{2}\right)-\frac{\pi(-1)^{n}\left(y^{2}\right)^{n-1} s^{n} \log \left(s / a^{2}\right)}{2^{2 n-2} n !(n-1) !}\right] .
$$

Comparison of $\left(7.7^{\prime}\right)$ and (7.13) yields

$$
F \delta_{a}^{(n)}\left(x^{2}\right)=\frac{-\pi\left(y^{2}\right)^{n-1}}{4^{n-1}(n-1) !}\left[\log \left|\frac{y^{2} a^{2}}{4}\right|-\Psi(n)-\Psi(n+1)\right] .
$$

(Note that "=" is a special case of " ".) Similarly we deduce from (7.9)

$$
F \varepsilon\left(x_{0}\right) \delta^{(n)}\left(x^{2}\right)=\frac{i \pi^{2} \varepsilon\left(y_{0}\right)\left(y^{2}\right)^{n-1}}{4^{n-1}(n-1) !}, \quad n \geqq 1 .
$$

Combining (7.14) and (7.15) we obtain

$$
\begin{aligned}
& F \theta\left(x_{0}\right) \delta_{a}^{(n)}\left(x^{2}\right) \\
& =\frac{-2 \pi\left(y^{2}\right)^{n-1}}{4^{n}(n-1) !}\left[\log \left|\frac{y^{2} a^{2}}{4}\right|-\Psi(n)-\Psi(n+1)-i \pi \varepsilon\left(y_{0}\right)\right], \quad n \geqq 1 .
\end{aligned}
$$




\subsection{Spectral Representations of Fourier Transforms}

Let $\theta\left(x_{0}\right) f\left(x^{2}\right)$ be given by the spectral representation

$$
\theta\left(x_{0}\right) f\left(x^{2}\right)=\int_{0}^{\infty} f(s) \theta\left(x_{0}\right) \delta\left(x^{2}-s\right) d s
$$

with $f(s) \in \mathscr{H}^{\prime}\left(R^{1}\right)$ and $f(s)=0$ for $s<0$. We find for the Fourier transform

$$
\begin{aligned}
\left\{F \theta\left(x_{0}\right) f\left(x^{2}\right)\right\}\langle\psi(y)\rangle & =(2 \pi)^{-4} \theta\left(x_{0}\right) f\left(x^{2}\right)\langle\varphi(-x)\rangle \\
& =(2 \pi)^{-4} \int_{0}^{\infty} f(s) \theta\left(x_{0}\right) \delta\left(x^{2}-s\right)\langle\varphi(-x)\rangle d s \\
& =\int_{0}^{\infty} f(s) F\left[\theta\left(x_{0}\right) \delta\left(x^{2}-s\right)\right] d s\langle\psi(y)\rangle .
\end{aligned}
$$

The Fourier operator obviously commutes with the (formal)s-integration and the assumption $f(s) \in \mathscr{H}^{\prime}$ is seen to be unessential since both $\theta\left(x_{0}\right) \delta\left(x^{2}-s\right)\langle\varphi(-x)\rangle$ and $F\left[\theta\left(x_{0}\right) \delta\left(x^{2}-s\right)\right]\langle\psi(y)\rangle$ are elements of $\mathscr{H}$. Thus we have with (2.20) the general representation of the Fourier transform of an invariant distribution $f(x) \in \mathscr{D}^{\prime}\left(R^{4}\right)$

$$
\begin{aligned}
F f(x)= & (2 \pi)^{3}\left[\int_{-\infty}^{\infty} f_{e h}(s) \Delta^{(1)}(y, s) d s-i \int_{0}^{\infty} f_{0}(s) \Delta(y, s) d s\right]+ \\
& +\sum_{n=0}^{N} c_{n}\left(y^{2}\right)^{n} .
\end{aligned}
$$

On the other hand, every invariant $f(y) \in \mathscr{Z}^{\prime}\left(C^{4}\right)$ admits the representation (7.17) where $f_{0}(s)$ is a uniquely determined element of $\mathscr{D}^{\prime}(s \geqq 0)$ and $f_{e h}(s)$ is a distribution from $\mathscr{H}^{\prime}\left(R^{1}\right)$. If we restrict the domain of $f(y)$ to the subspace $\mathscr{Z}_{0}\left(C^{4}\right), f_{e}(s)$ is a uniquely determined distribution of $\mathscr{D}^{\prime}\left(R^{1}\right)$. (7.17) has the structure of the KäLLEN-LEHMANN representation if $y$ is interpreted as a vector of the space-time continuum. Since $f(y)$ is considered as an element of $\mathscr{Z}^{\prime}\left(C^{4}\right)$ strongly increasing spectral functions $f(s)$ are admissible.

If $f_{e}(s)$ and $f_{0}(s)$ are functions with an algebraic singularity at $s=0$ the most convenient form of (7.17) is given by

$$
\begin{aligned}
F f_{a}(x)= & \operatorname{Res}_{z=0}\left\{\frac { ( 2 \pi ) ^ { 3 } } { z } \left[\int_{-\infty}^{\infty} f_{e}(s)\left(\frac{a^{2}}{s}\right)^{z} \Delta^{(1)}(y, s) d s-\right.\right. \\
& \left.\left.-i \int_{0}^{\infty} f_{0}(s)\left(\frac{a^{2}}{s}\right)^{z} \Delta(y, s) d s\right]\right\}
\end{aligned}
$$

(The vanishing of the additional polynomial is due to the definition of $\left.f_{a}(x).\right)$ 
Let us finally observe that the spectral representation in $x$ and $y$-space of an odd invariant distribution is a special case of the Jost-LenmanNDyson representation of a local commutator with prescribed support properties in momentum space. Thus (7.17) may perhaps be extended into a subtraction-free generalization of the JLD representation.

\section{\%.4. Fourier Transforms of Power Type Distributions}

To illustrate the power of invariant spectral representations let us consider a few representative examples of Fourier transforms.

According to the preceding section we obtain for $F\left[\theta\left(x_{0}\right)\left(x^{2}\right)^{\lambda}\right]_{a}$ the expression

$F\left[\theta\left(x_{0}\right)\left(x^{2}\right)^{\lambda}\right]_{a}=\operatorname{Res}_{z=0}\left\{\frac{-i \pi^{2}}{z} \int_{0}^{\infty} \frac{s^{\lambda+1}}{\sqrt{\xi^{2}}}\left(\frac{a^{2}}{s}\right)^{z} H_{1}^{(1)}\left(\sqrt{s \xi^{2}}\right) d s\right\}_{\xi^{3}=y^{2}+i y 0}$

We have first to evaluate the $s$-integral in a $z$-region where the $r h s$ of (7.18) is analytic in $z$ (and therefore $a$-independent). Performing the well known Hankel transformation we immediately arrive at

$$
F\left[\theta\left(x_{0}\right)\left(x^{2}\right)^{\lambda}\right]_{a}=8 \pi 4^{\lambda} e^{i \pi \lambda} \Gamma(\lambda+1) \Gamma(\lambda+2)\left(y^{2}+i y_{0} 0\right)^{-\lambda-2}
$$

for $\lambda \neq-1,-2,-3, \ldots$. Similarly we obtain $(\lambda \neq-1,-2, \ldots)$

$$
\begin{aligned}
F\left[\theta\left(-x^{2}\right)\left|x^{2}\right|^{\lambda}\right]_{a}= & -\pi 4^{\lambda+2} \Gamma(\lambda+1) \Gamma(\lambda+2)\left[\theta\left(y^{2}\right)\left(y^{2}\right)^{-\lambda-2}+\right. \\
& \left.+\theta\left(-y^{2}\right)\left|y^{2}\right|^{-\lambda-2} \cos \lambda \pi\right] .
\end{aligned}
$$

In order to get the Fourier transform at $\lambda=-n$ we have to compute the $\lambda$-free terms in the Laurent expansions of (7.19) and (7.20) around the point $\lambda=-n$. This gives us the Fourier transform for $a=1$. The $a$-dependent Fourier transform is then easily obtained by formula (7.6). With

we find

$$
\begin{aligned}
\Gamma(-m+z)= & \frac{(-1)^{m}}{m !}\left[z^{-1}+\Psi(m+1)+\frac{1}{2}\left(\Psi^{2}(m+1)-\right.\right. \\
& \left.\left.-\Psi^{\prime}(m+1)+\frac{\pi^{2}}{3}\right) z+\cdots\right]
\end{aligned}
$$

$$
\begin{aligned}
F\left[0\left(x_{0}\right)\left(x^{2}\right)^{-1}\right]_{a}= & -4 \pi \Psi(1)\left(y^{2}+i y_{0} 0\right)^{-1}+2 \pi\left(y^{2}\right)_{1 / a}^{-1} \log \frac{y^{2} a^{2}}{4}- \\
& -2 \pi^{2} i \varepsilon\left(y_{0}\right)\left(y^{2}\right)_{1 / 2 a}^{-1}-\pi^{3} \delta\left(y^{2}\right), \\
F\left[0\left(-x^{2}\right)\left|x^{2}\right|^{-1}\right]_{a}= & 4 \pi\left(y^{2}\right)_{1 / 2 a}^{-1}\left[\log \left|\frac{y^{2} a^{2}}{4}\right|-2 \Psi(1)\right]+2 \pi^{3} \delta\left(y^{2}\right) .
\end{aligned}
$$

For $n=2,3,4, \ldots$ we have

$$
\begin{aligned}
& F\left[\theta\left(x_{0}\right)\left(x^{2}\right)^{-n}\right]_{a} \\
& =\frac{8 \pi(-1)^{n-1}\left(y^{2}\right)^{n-2}}{4^{n}(n-1) !(n-2) !}\left[K_{1}+K_{2} \log \left(\frac{\left.-y^{2}-i y_{0} 0\right) a^{2}}{4}\right)+\right. \\
& \left.\quad+\frac{1}{2} \log ^{2}\left(\frac{\left(-y^{2}-i y_{0} 0\right) a^{2}}{4}\right)\right]
\end{aligned}
$$


where

$$
\begin{aligned}
K_{1}= & \Psi(n-1) \Psi(n)+\frac{1}{2}\left[\Psi^{2}(n)-\Psi^{\prime}(n)+\frac{\pi^{2}}{3}\right]+ \\
& +\frac{1}{2}\left[\Psi^{2}(n-1)-\Psi^{\prime}(n-1)+\frac{\pi^{2}}{3}\right], \\
K_{2}= & -\Psi(n)-\Psi(n-1) ; \log \left(-y^{2}-i y_{0} 0\right)=\log \left|y^{2}\right|-i \pi \varepsilon\left(y_{0}\right) .
\end{aligned}
$$

Similarly

$$
\begin{aligned}
& F\left[\theta\left(-x^{2}\right)\left|x^{2}\right|-n\right]_{a} \\
&= \frac{16 \pi\left(y^{2}\right)^{n-2}}{4^{n}(n-1) !(n-2) !}\left[K_{1}+K_{2} \log \left|\frac{y^{2} a^{2}}{4}\right|+\frac{1}{2} \log ^{2}\left|\frac{y^{2} a^{2}}{4}\right|\right]- \\
& \quad-\frac{8 \pi^{3}(-1)^{n} \theta\left(-y^{2}\right)\left|y^{2}\right|^{n-2}}{4^{n}(n-1) !(n-2) !} .
\end{aligned}
$$

Combining (7.24) and (7.25) we arrive at

$$
F\left(x^{2}\right)^{-n}=\frac{8 \pi^{3}(-1)^{n} \varepsilon\left(y^{2}\right)\left(y^{2}\right)^{n-2}}{4^{n}(n-1) !(n-2) !} .
$$

We see that the Fourier operator transforms an associated function into an associated function of the same order and that these generalized functions are dimensionally correct only if $a$ has the same dimension as $x$ or $1 / y$.

Further formulas will be listed in [10].

Acknowledgments. The authors wish to express their sincere gratitude to Dr. E. Pfaffelhuber for many helpful discussions and to Prof. F. Bopp for his interest in this work. Particular thanks are due to the Deutsche Forschungsgemeinschaft for financial support.

\section{References}

1. Schwartz, L.: Theorie des distributions, I, II. Paris: Hermann 1950/51.

2. Methée, P. D.: Commun. Math. Helv. 28, 225 (1954); Coll. CNRS, Nancy 1956.

3. Braga, C.: Univ. S. Paulo Preprint 1960.

4. Garding, L., e J. L. Lions: Nuovo Cimento Vol. XIV, Ser. X (1959).

5. Gelfand, I. M., and G. E. Shrlov: Generalized functions I. New York, London: Academic Press 1964.

6. Güttinger, W.: Fortschr. Physik 14, 483 (1966).

7. Pfaffelhuber, E.: Univ. München, Thesis (1966).

8. Güttinger, W., and E. Pfaffelhuber: Nuovo Cimento 52, 389 (1967).

9. Rohrlich, F., e C. Gorá́: Univ. Syracuse Preprints (1966).

10. Güttinger, W., E. Pfaffelhuber u. A. Rieckers: Univ. München, Preprint (1967).

11. Cf., e.g., Schweber, S.: Introduction to relativistic quantum field theory. New York: Harper \& Row 1961.

Prof. W. Güttinger

Sektion Physik

Universität München

8000 München 13

Schellingstr. 2-8 